\title{
REVIEW
}

\section{Photogeneration of hot plasmonic electrons with metal nanocrystals: Quantum description and potential applications}

\author{
Alexander O. Govorov ${ }^{a, *}$, Hui Zhang ${ }^{a}$, Hilmi Volkan Demir ${ }^{b, c}$, \\ Yurii K. Gun'kod,e
}

\author{
a Department of Physics and Astronomy, Ohio University, Athens, OH 45701, USA \\ b Department of Electrical and Electronics Engineering, Department of Physics, UNAM - Institute of \\ Materials Science and Nanotechnology, Bilkent University, Ankara 06800, Turkey \\ c School of Electrical and Electronic Engineering, School of Physical and Mathematical Sciences, Nanyang \\ Technological University, Nanyang Avenue, Singapore 639798, Singapore \\ d School of Chemistry and CRANN, University of Dublin, Trinity College, Dublin 2, Ireland \\ e Saint Petersburg National Research University of Information Technologies, Mechanics and Optics, 197101 \\ Saint Petersburg, Russia
}

Received 5 November 2013; received in revised form 20 January 2014; accepted 4 February 2014

\author{
KEYWORDS \\ Plasmon; \\ Plasmonic electrons; \\ Injection of \\ electrons; \\ Nanostructures; \\ Photoelectric effect; \\ Photodetectors; \\ Photocatalysis
}

\begin{abstract}
Summary The paper reviews physical concepts related to the collective dynamics of plasmon excitations in metal nanocrystals with a focus on the photogeneration of energetic carriers. Using quantum linear response theory, we analyze the wave function of a plasmon in nanostructures of different sizes. Energetic carriers are efficiently generated in small nanocrystals due to the non-conservation of momentum of electrons in a confined nanoscale system. On the other hand, large nanocrystals and nanostructures, when driven by light, produce a relatively small number of carriers with large excitation energies. Another important factor is the polarization of the exciting light. Most efficient generation and injection of high-energy carriers can be realized when the optically induced electric current is along the smallest dimension of a nanostructure and also normal to its walls and, for efficient injection, the current should be normal to the collecting barrier. Other important properties and limitations: (1) intra-band transitions are preferable for generation of energetic electrons and dominate the absorption for relatively long wavelengths (approximately $>600 \mathrm{~nm}$ ), (2) inter-band transitions efficiently generate energetic holes and (3) the carrier-generation and absorption spectra can be significantly different.
\end{abstract}

\footnotetext{
* Corresponding author. Tel.: +1 7405939430.

E-mail address: govorov@ohiou.edu (A.O. Govorov).
} 
The described physical properties of metal nanocrystals are essential for a variety of potential applications utilizing hot plasmonic electrons including optoelectronic signal processing, photodetection, photocatalysis and solar-energy harvesting.

(C) 2014 Elsevier Ltd. All rights reserved.

\section{Introduction}

Whereas plasmonic properties of metal nanocrystals in terms of optical response have been so intensively investigated $[1,2]$, the internal quantum states of photo-excited electrons inside plasmonic systems are much less known because electrons in nanocrystals oscillate in a nontrivial way, creating a collective excitation, a plasmon. Under optical illumination, the electrons simultaneously form a plasmon excitation and scatter by each other and from the walls and phonons. Experimentally, optically excited electrons in a metal can be registered via a photocurrent in a semiconductor-metal Schottky-barrier photodetector [3] (Fig. 1a) or using surface photochemistry [4,5] (Fig. 1b). It has been recognized recently that plasmonic nanostructures and nano-antennas can be used for hot carrier generation, photocatalysis, and injection. Metal nanocrystals have large absorption cross sections and can efficiently enhance and trap light [6-8]. Plasmonic enhancement of photocurrents and chemical processes can be induced via direct electron transfer from a metal or indirectly via a local amplification of electromagnetic field at functional elements of a device [9]. Several recent papers reported plasmon-enhanced photochemistry [9-22] (when plasmonic nanocrystals are in contact with a liquid) and plasmon-driven photocurrent responses in optoelectronic devices and nanostructures [23-33].

The photo-excited plasmonic electrons look very attractive for applications in photochemistry, solar cells, and photodetectors because metal nanocrystals can absorb light much more efficiently compared to inorganic semiconductors and organic dye molecules. Here are some advantages of metal nanocrystals:

- Large absorption cross sections.

- A large number of electrons with appropriate energy levels for electron transfer.

- Efficient tuning of the plasmonic resonances, absorption spectra and local enhancement using the size, shape, orientation and arrangement of nanocrystals [1,2,34-36].

Fig. 2 illustrates gold nanocrystals of various shapes and shows their absorption spectra. We can see that the position of plasmon resonance in absorption can be conveniently tuned with the shape of a nanocrystal. However, there are significant fundamental limitations for the use and extraction of plasmonic electrons in photocatalytic and solar conversion applications. These limitations include:

- Fast relaxation and relatively short mean free path of electrons in metals.

- Limited momentum transfer and often a large number of excited electrons with low energies.

- Reflection of electrons from the metal-semiconductor interface.
In this perspective article, we will address physical properties of plasmons in metal nanocrystals, focusing on the microscopic quantum structure of plasmonic oscillations and the non-equilibrium electron distributions in optically driven confined systems. The first sections will describe energy distributions and dynamics of excited electrons in the plasmon waves in the bulk and in confined nanocrystals. The following sections will address model physical systems and devices as well as provide a brief review of experimental work in the field.

\section{Wave functions of plasmons in the bulk and in nanostructures}

\section{D metal}

Electrons in metals form a Fermi gas, which is characterized by the Fermi velocity $\left(v_{F}\right)$, Fermi energy $\left(E_{F}\right)$, and bulk plasmon frequency, $\left(\omega_{p, b u l k}\right)$. The non-interacting Fermi-gas model gives the following equations for the above parameters $[38,39]$

$E_{F}=\frac{m_{0} v_{F}^{2}}{2}=\frac{{ }^{2}\left(3 \pi^{2} n_{0}\right)^{2 / 3}}{2 m_{0}}, \omega_{p, \text { bulk }}=\sqrt{\frac{4 \pi e^{2} n_{0}}{\varepsilon_{0} m_{0}}}$,

where $n_{0}$ is the $3 \mathrm{D}$ electron density, $m_{0}$ is the electron mass and $\varepsilon_{0}$ is the background dielectric constant coming from the atomic core electrons. For gold, these numbers are $E_{F}=5.5 \mathrm{eV}, v_{F}=1.39 \times 10^{8} \mathrm{~cm} / \mathrm{s}$, and $\omega_{p, \text { bulk }}=3.96 \mathrm{eV}[40]$. The quantum description of electron gas is based on the density matrix, $\rho_{m n}$ [38]. The elements of the density matrix give us populations of quantum single-particle states in an electron gas driven by optical excitation. The population of a single-particle quantum state in a Fermi gas is simply the corresponding matrix element

$\rho_{n n}(t)=\left\langle\Psi(t)\left|\hat{c}_{n}^{+} \hat{c}_{n}\right| \Psi(t)\right\rangle$,

where $|\Psi(t)\rangle$ is a non-equilibrium wave function, which may be quite complicated in its exact form, and the operators $\hat{c}_{n}^{+}$and $\hat{c}_{n}$ are the creation and annihilation operators, respectively. Then, the operator $\hat{c}_{n}^{+} \hat{c}_{n}$ "probes", the many-body wavefunction $|\Psi(t)\rangle$ and reveals the presence of equilibrium or non-equilibrium electrons in the Fermi sea. In the absence of illumination, the system is in equilibrium and the population is, of course, given by the Fermi distribution function $\rho_{n n}=f_{F}(E)$, where $E$ is the electron energy. When the electron plasma is illuminated, the non-diagonal elements, like $\rho_{n^{\prime} n}(t)=\left\langle\Psi(t)\left|\hat{c}_{n}^{+} \hat{c}_{n^{\prime}}\right| \Psi(t)\right\rangle$, reveal the presence of an excitation, an electron-hole pair, in the Fermi gas (Figs. 3b and $4 a$ ). In the election-hole excitation, an electron is promoted from the state $|n\rangle$ 
(a)
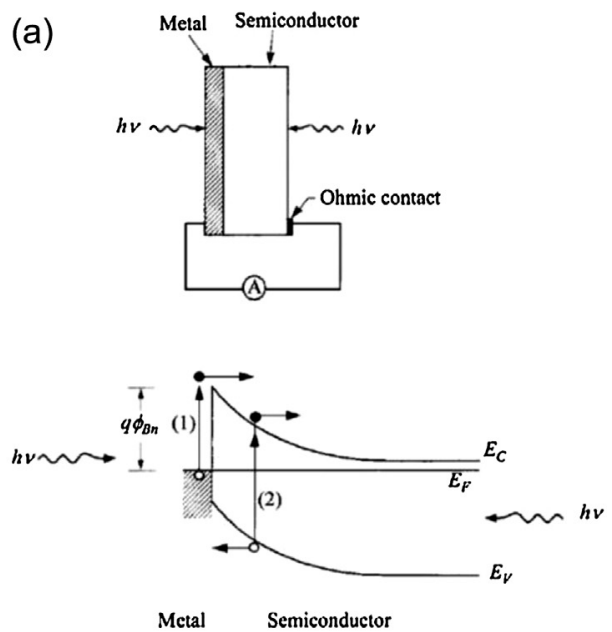

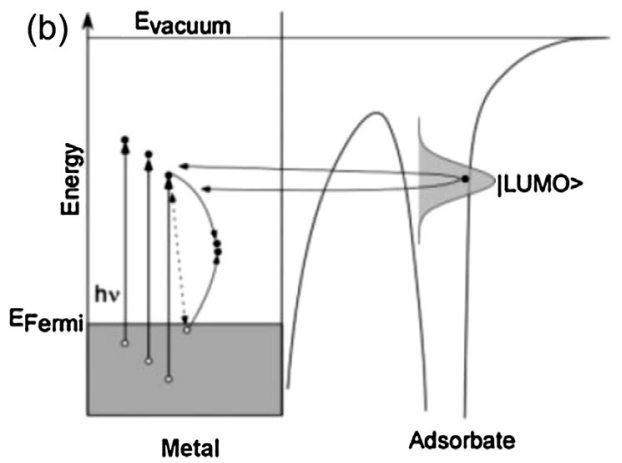

Metal
Adsorbate

Figure 1 (a) Schematic setup for photoelectric experiment with a metal-semiconductor Schottky contact and the related energyband diagram for the photoexcitation processes. Reprinted with permission from Ref. [3]. Copyright $\odot 2007$ by John Wiley \& Sons, Inc. (b) Schematic illustration of hot-electron transfer from a metal to an unoccupied molecular orbital at the surface. The laser light generates hot electrons in the bulk and these electrons have a finite probability to tunnel to the adsorbate. Reprinted with permission from Ref. [4]. Copyright (2013) American Chemical Society.

below the Fermi level to the non-equilibrium state $\left|n^{\prime}\right\rangle$ above the Fermi-sea level (Figs. 3b and 4a). The density matrix describes the quantum state of electron gas and should be found from the solution of the equation of motion $[38,41]$

$$
\frac{\partial \hat{\rho}}{\partial t}=i[\hat{\rho}, \hat{H}(t)]-\hat{\Gamma}_{r e l} \hat{\rho}
$$

\section{(a)}

\section{Tailoring surface plasmon resonances in} nanocrystals
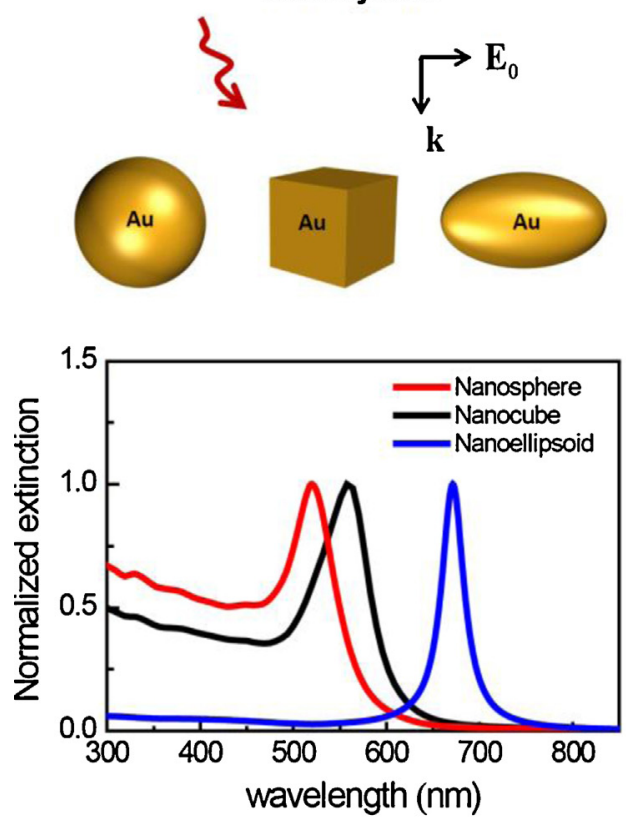

(b)
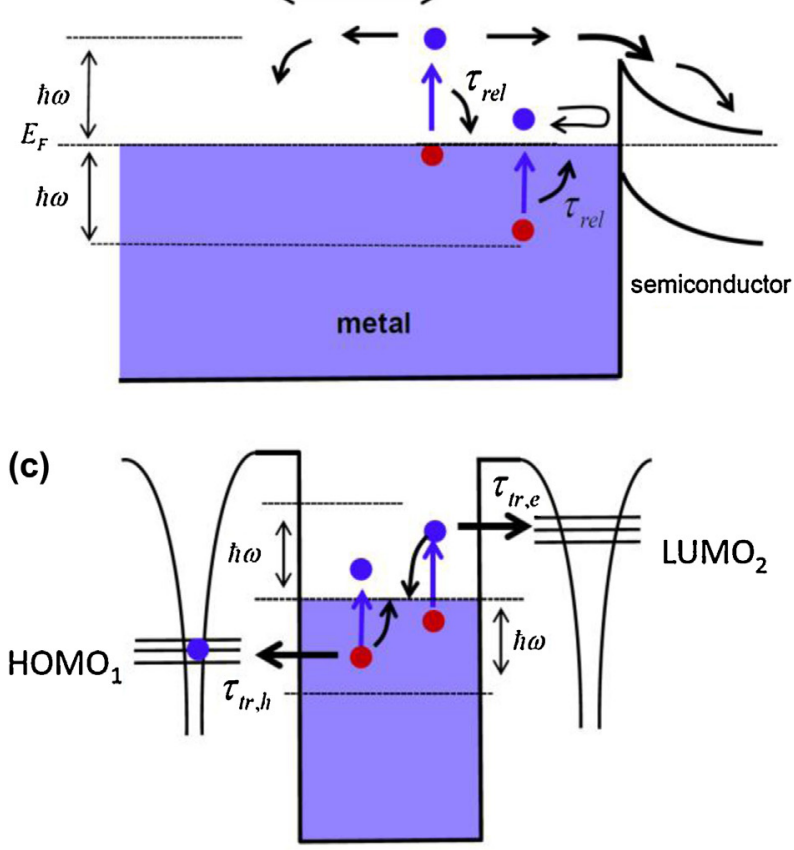

adsorbate metal adsorbate

Figure 2 (a) Schematic illustration of gold nanocrystals and their absorption spectra in water. The absorption spectra were calculated numerically using the local dielectric constant of gold. [37] (b) Physical processes in a photo-excited electron gas that is in contact with a semiconductor. (c) Optoelectronic processes in a confined nanocrystal in contact with molecular adsorbate. Photogenerated plasmonic carriers have chance to be transferred to the molecules adsorbed on the surface. 
(a)

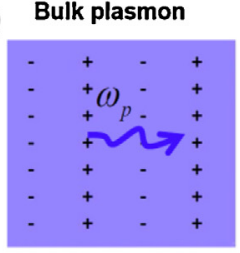

(b)

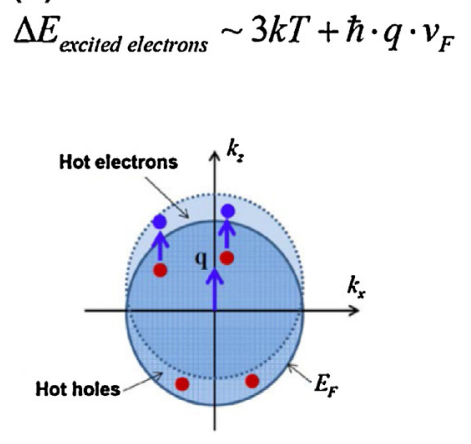

(c)

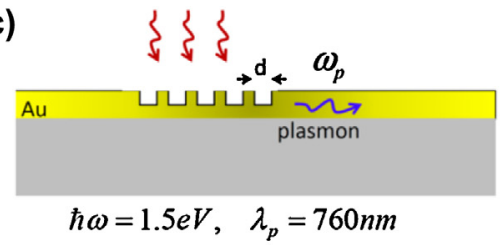

(d)

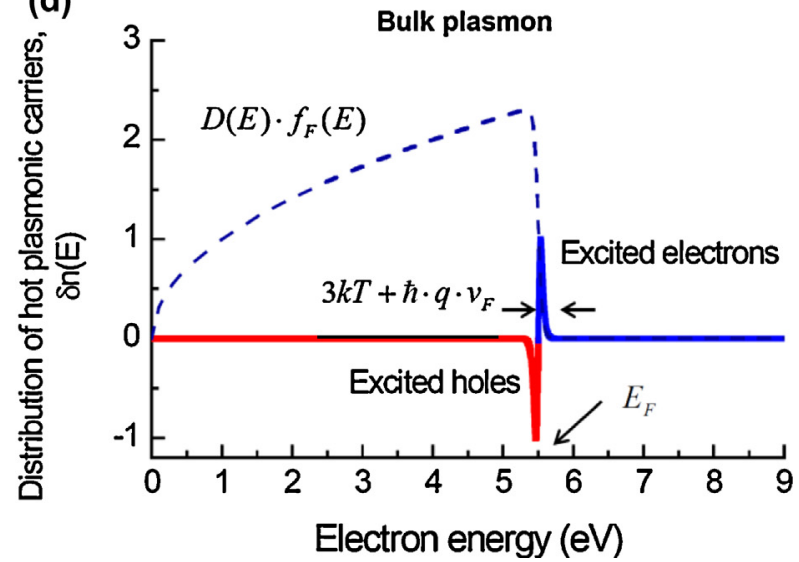

Figure 3 (a) and (b) Schematic illustrations for a propagating plasmon wave in a bulk crystal and the Fermi sea of electrons in the bulk with a plasmon excitation. (c) Surface plasmon polariton in an Au waveguide. (d) Plasmonic electron distribution in the bulk plasmon. The positive (blue) part represents the population of electrons above the Fermi energy while the negative (red) part is the hole population below the Fermi energy. The Fermi energy $E_{F}=5.5 \mathrm{eV}$, which corresponds to the case of bulk gold. The dashed curve shows the equilibrium Fermi distribution of electrons at room temperature.

(a) $\Delta E_{\text {excited electrons }} \sim \hbar \omega$

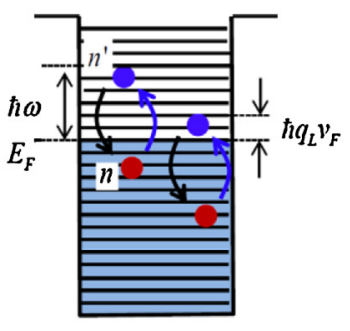

(c)

$\hbar \omega=2.22 \mathrm{eV}$

(b)
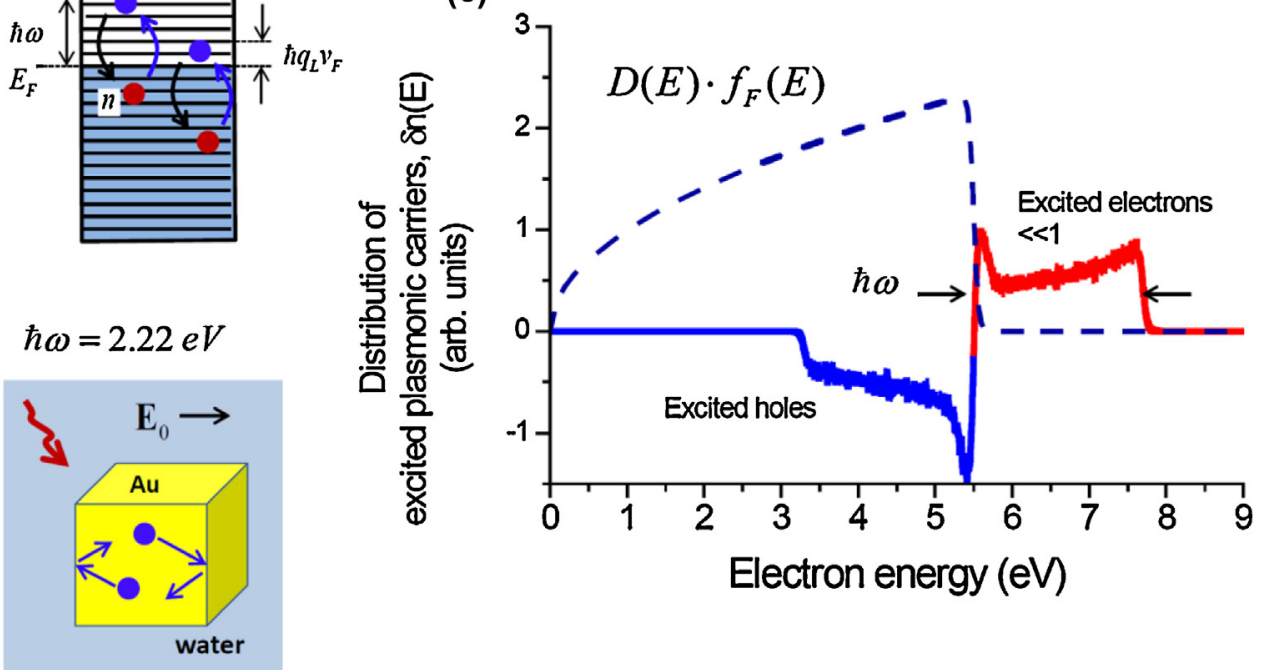

Figure 4 (a) Schematics of electron transitions in a metal nanocrystal. A Fermi-sea electron becomes first optically excited and then undergoes energy relaxation. In this way, a steady-state distribution of high-energy electrons in a localized plasmon wave becomes formed; $q_{L}=\pi / L_{N C}$ is the characteristic change of the momentum of the electron and $q_{L} v_{F}$ is the elementary excitation energy of an electron at the Fermi level in a nanocrystal due to the non-conservation of linear momentum. (b) Model of an $\mathrm{Au}$ nanocube with electrons bouncing off the walls. (c) Plasmonic electron distribution in the localized plasmon in a $10 \mathrm{~nm}$ gold nanocube. The dashed curve shows the equilibrium Fermi distribution of electrons. The data for the nanocube were obtained numerically from Eq. (3). For the moderate light intensities typically used in the experiments, the non-equilibrium population of energetic carriers is much smaller that the equilibrium Fermi distribution. 
where $\hat{H}$ is the Hamiltonian including the optical excitation and $\hat{\Gamma}_{\text {rel }}$ is an operator describing energy relaxation. The equation of motion in the form (1) provides us with a very convenient approach for treating many quantum systems $[38,39,41]$.

We start with a plasmon wave in the bulk and in the systems with large dimensions (Fig. $3 a$ and $\mathrm{c}$ ). The dynamic response of the Fermi gas in the presence of the external field is described by the bulk dielectric function [38]:

$\varepsilon_{3 D}(\omega, q)=1-\frac{4 \pi e^{2}}{q^{2} V} \sum_{k} \frac{f_{F}(\mathbf{k})-f_{F}(\mathbf{k}+\mathbf{q})}{\omega-E_{\mathbf{k}+\mathbf{q}}+E_{\mathbf{k}}+i \gamma_{\text {rel }}}$

where $\mathbf{q}$ is wave vector of excitation in the gas and $V$ is the crystal volume. The zeros of this function should give self-oscillations of the system. Indeed one can show that the equation $\varepsilon_{3 D}(\omega)=0$ produces the plasmon frequency $\omega=$ $\pm \omega_{p, \text { bulk }}$.

Now we look at the structure of a plasmon wave, i.e. the electron distribution. The steady-state distribution of hot plasmonic electrons can be obtained directly from the diagonal elements

$\delta n(E)=2 \cdot \sum_{n} \delta \rho_{n n} \cdot \delta\left(E-E_{n}\right)$

where $\delta\left(E-E_{n}\right)$ is a numerical $\delta$-function, $E_{n}$ is the energy of the singe-particle state $|n\rangle$, and the factor 2 accounts for spins. The equation for the time-averaged function $\delta \rho_{n n}$, derived from the linear response theory in Ref. [42], reads:

$$
\begin{aligned}
\delta \rho_{n n}= & \frac{2}{\gamma_{r e l}} \sum_{n^{\prime}}\left(f_{n^{\prime}}^{0}-f_{n}^{0}\right)\left|e \varphi_{n n^{\prime}}\right|^{2}\left[\frac{\gamma_{r e l}}{\left(\omega-E_{n}+E_{n^{\prime}}\right)^{2}+\gamma_{r e l}{ }^{2}}\right. \\
& \left.+\frac{\gamma_{r e l}}{\left(\omega+E_{n}-E_{n^{\prime}}\right)^{2}+\gamma_{r e l^{2}}}\right] .
\end{aligned}
$$

where

$$
\varphi_{n n^{\prime}}=\left\langle n\left|\varphi_{\omega}(r)\right| n^{\prime}\right\rangle
$$

is the matrix elements of optical excitations, $\varphi_{\omega}(r)$ is a complex amplitude of the time-oscillating electric potential induced by the illumination and $\gamma_{\text {rel }}$ is the energy relaxation rate of electrons. These matrix elements produce quantum amplitudes of optical transitions between the electron states. Fig. 3d shows the calculated hot plasmonic distributions in a bulk plasmon taken from Ref. [42]. We assume that the plasmon wave excited in an electron gas has the wavelength $\lambda=760 \mathrm{~nm}$. The excited-electron distribution $\delta n(E)=n(E)-f_{F} \cdot D(E)$ is positive for $E>E_{F}$ and negative for $E<E_{F}$; here $D(E)$ is the electron density of states of the bulk system. The function $\delta n(E)$ reveals the presence of electron-hole pair excitations in the optically driven electron Fermi sea. Because of the conservation of momentum in the bulk, the transitions occur only between the states $|n\rangle=|\mathbf{k}\rangle$ and $\left|n^{\prime}\right\rangle=|\mathbf{k}+\mathbf{q}\rangle$, where $\mathbf{q}$ is the wavevector of the bulk plasmon. Consequently, the sum (3) includes only the transitions between states $|\mathbf{k}\rangle$ and $|\mathbf{k}+\mathbf{q}\rangle$ that have small excitation energies $\Delta E=E_{\mathbf{k}+\mathbf{q}}+E_{\mathbf{k}} \sim q v_{F}$. The energy $q v_{F}$ is small and typically less than the plasmon and ther- mal energies, $q v_{F}<k_{B} T \ll \omega_{p, b u l k}$. For a model system with the Fermi energy of gold at room temperature, which has $\omega_{p, b u l k}=3.96 \mathrm{eV}$ and $k_{B} T=0.026 \mathrm{eV}$, the excitation energy $q v_{F}=0.007 e V$ assuming a plasmon wave with a wavelength of $760 \mathrm{~nm}$. Therefore, the typical energy of an excited carrier counted with respect to the Fermi energy in the plasmon wave

$\Delta E_{\text {excited electrons }} \sim 3 k_{B} T+q v_{F}=0.08 \mathrm{eV}$

under the usual condition $q \ll k_{F}$. We see an interesting property of the bulk plasmon wave: Electrons in the plasmon with a large energy quantum $\left(\omega_{p, b u l k} \sim 3 e V\right)$ have very small excitation energies of single electrons. In many cases, it is more convenient to perform plasmonic experiments using a planar waveguide (Fig. 3c) [1,2] where plasmons with optical energies $(\omega \sim 1-2 e V)$ can be launched and then detected at different lateral positions.

\section{Plasmons in a waveguide}

Coupled plasmon-electromagnetic waves in a waveguide are called Surface Plasmon Polaritons (SPPs). For example, a SPP in a Au waveguide with a wavelength $760 \mathrm{~nm}$ has the energy quantum $\omega_{\text {SPP }} \sim 1.5 \mathrm{eV}$ [43]. To launch a SPP wave, one can use a grating (Fig. 3c) $[1,43]$. The width of the metal waveguide is typically large $(\sim 150 \mathrm{~nm}$ in Ref. [43]), much longer than the mean-free-path of an electron. Therefore, we can use the bulk theory to understand the electronic structure of a SPP wave. Estimating the excitation energy with Eq. (4), we see again that SPPs with a realistic wavelength create mostly very low-energy electron excitations, $\Delta E_{\text {excited electrons }} \sim 3 k_{B} T \sim 0.08 \mathrm{eV} \ll \omega_{S P P}=1.5 \mathrm{eV}$. We, therefore, conclude that plasmonic systems with large dimensions have the serious limitation for efficient electron photo-injection into a semiconductor since the excited plasmonic electrons need high enough energy to pass the Schottky barrier. In other words, it is expected that SPPs launched in a waveguide cannot create many high-energy electrons, which can be detected by a Schottky barrier with a typical height of $0.5-1 \mathrm{eV}$. Nevertheless, the experiment with an $\mathrm{Au}-\mathrm{Si}$ photo-detector has shown injected currents. [24] The physical explanation for this observation can be in breakdown of momentum conservation of electrons due to defects, phonons, and interfaces. This simple argument suggests that the size of metal nanocrystal can be very important for the plasmonic generation of electrons with large excitation energies. We will describe this physical behavior in the next section.

\section{Plasmonic nanocrystals (NCs)}

\section{General remarks}

The picture of electron excitations in a confined electron gas is dramatically different [42]. The physical reason is the non-conservation of electron momentum due to potential walls of the nanocrystal. Fig. 4 illustrates this case. The energy of electrons is quantized and optical transitions occur between the discrete states (Fig. 4a). The distribution of excited electrons in a localized plasmon is very different from the case of bulk-like system (Fig. 3d). The excited 
(a)

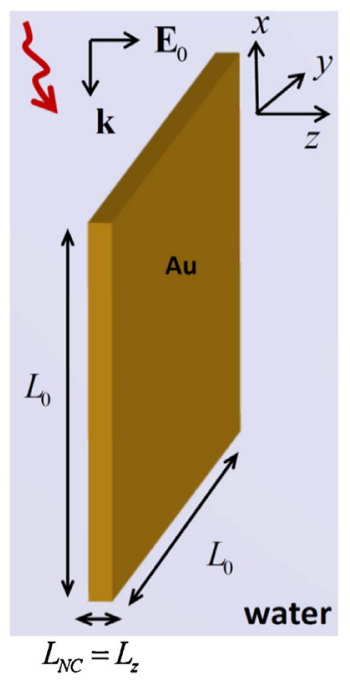

(b)

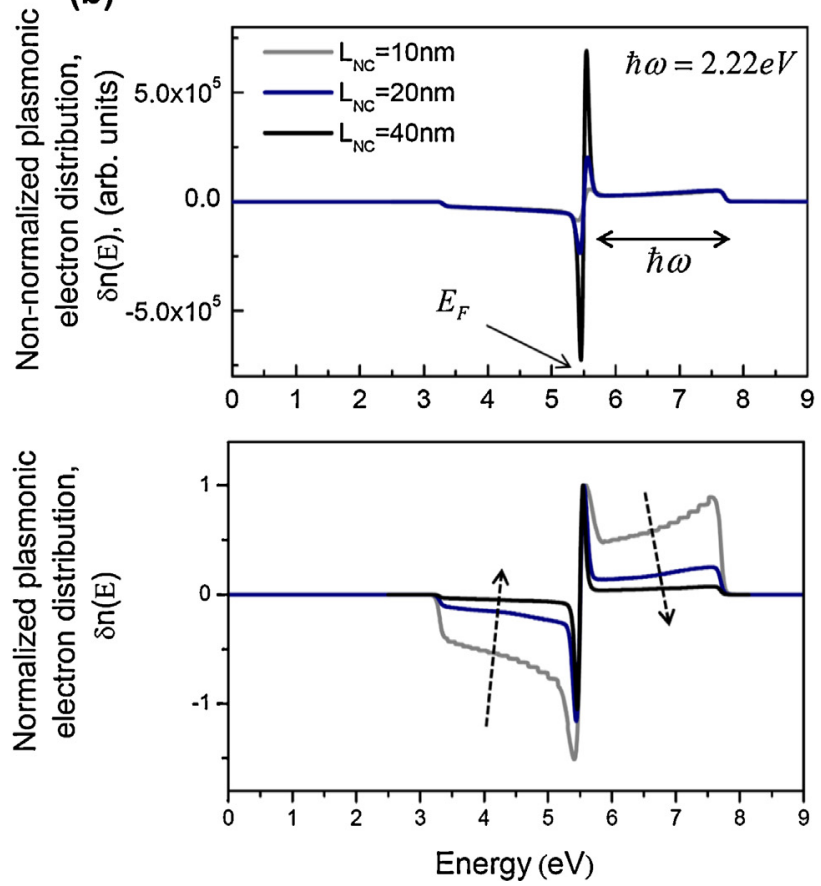

Figure 5 (a) Schematic illustration of a plasmonic Au slab in water driven by the electric field of incident light. (b) Distributions of plasmonic carriers in the slabs with a variable width. The exciting electric field of incident illumination is normal to the slab walls. The positive region of $\delta n(E)$ describes excited electrons in the Fermi sea, whereas the negative part indicates the presence of excited holes. For convenience, we show both non-normalized and normalized distributions. The upper graph shows that the flat parts of the distribution function do not depend on the slab width. This indicates that the photogeneration in these parts is due to the surface scattering of carriers.

electrons in a confined nanocrystal occupy the whole region of the allowed energies [42]

$E_{F}<E_{\text {excited electrons }}<E_{F}+\omega$.

This can be understood using the following arguments. Because of the conservation of energy, an excited electron can acquire the energy $E_{\text {excited }}=E+\omega$. Therefore, the maximum allowed energy of the electron in the system is $E_{\max }=E_{F}+\omega$ and, as we see, the excited-electron distribution extends from the Fermi energy to the maximum value (the blue part of the curve in Fig. 4c). Correspondingly, the energy interval for photo-excited holes is $E_{F}-\omega<$ $E_{\text {excited holes }}<E_{F}$ (the red part on the curve in Fig. $4 \mathrm{c}$ ).

\section{Plasmonic slab}

As the simplest example, we consider now a plasmonic platelet (slab) with the smallest dimension $L_{N C}$ along the $z$-axis (Fig. 5a). The incident electromagnetic wave is polarized in the $z$-direction. This dynamic electric field creates oscillating electric currents that hit the walls of the slab. Therefore, the Fermi gas becomes strongly perturbed and we can expect a significant number of electrons with large energies. The electron distribution calculated by using the theory developed in Ref. [42] confirms our expectation (Fig. 5b). Moreover, the number of highly excited electrons is large only for relatively small sizes $L_{N C}$. With increasing $L_{N C}$, the number of electrons with large excitation energies decreases rapidly and already for $L_{N C}=40 \mathrm{~nm}$ the electron distribution resembles the bulk plasmon (Fig. 5b). To qualitatively explain this behavior, we look now at the electron excitation of a quantized system. In a nanocrystal with a quantized motion along the $z$-direction, the electrons spectrum takes the form of

$E_{n, k_{\|}}=\frac{{ }^{2} \pi^{2} n^{2}}{2 m_{0} L_{N C}^{2}}+\frac{{ }^{2} k_{\|}^{2}}{2 m_{0}}$,

where $n$ is the quantum number for the $z$-motion and $\mathbf{k}_{\|}$ is the electron momentum in the plane of a slab. A photon excites an electron from the state $|n\rangle$ to the state $\left|n^{\prime}\right\rangle$ and the change in the electron number can only be odd, $\Delta n=1,3,5, \ldots$, due to the parity. Then, the characteristic energy of electron excitations in a confined system is given by the equation

$$
\begin{aligned}
& \Delta E=E_{n+\Delta n}+E_{n} \approx \frac{{ }^{2} \pi^{2} n \cdot \Delta n}{m_{0} L_{N C}^{2}} \sim v_{F}\left(\frac{\pi \Delta n}{L_{N C}}\right), \\
& \Delta n=1,3,5, \ldots,
\end{aligned}
$$

assuming $\pi \Delta n / L_{N C}<k_{F}$ and that all optical transitions occur in the vicinity of the Fermi sea: $E_{n, \mathbf{k}_{\|}} \approx E_{F}$ or $0<$ $(\pi n) /\left(L_{N C} m_{0}\right) \leq v_{F}$. Importantly, the effective momentum transfer, which comes from the electron confinement, can be significant and is given by

$\Delta k=\left(\frac{\pi \Delta n}{L_{N C}}\right)=\Delta n \cdot k_{L}$ 
where $\Delta n$ can be a large number and $k_{L}=\pi / L_{N C}$ is the characteristic change of electron momentum due to the NC confinement. Since $\Delta n$ can be large, this momentum transfer from the nanocrystal to single electrons permits efficient excitation of electrons with energies in the whole interval $E_{F}<E_{\text {excited electrons }}<E_{F}+\omega$ (Fig. $4 c$ and $5 \mathrm{~b}$ ). The excitation energies of single electrons are now in the optical range, $1-3 \mathrm{eV}$, and such electrons can be used for chemical reactions at the surface or for efficient injection into a semiconductor. In the next two sections, we will discuss such applications. Due to the conservation of energy, the most important momentum transfer numbers $\Delta n$ should be in the interval $\Delta n>\Delta n_{\text {critical }}$, where the critical number is given by the condition [42]

$v_{F}\left(\frac{\pi \Delta n_{\text {critical }}}{L_{N C}}\right)=\omega$

The effect of generation of high-energy electrons is strong if the above number (i.e. $\Delta n_{\text {critical }}$ ) is not too large [42]. The above consideration is also applied to the hot plasmon holes generated in the interval $E_{F}-\omega<$ $\Delta E_{\text {excited holes }}<E_{F}$. Some other properties of the function $\delta n(E)$ can be seen from this equation for the plasmonic slab taken from Ref. [42]:

$\delta n(E) \sim \frac{A}{\gamma_{\text {rel }}}\left|E_{z, \text { inside }}\right|^{2}$ for $E_{F}+3 k_{B} T+q v_{F}<E<E_{F}+\omega$

where $\gamma_{\text {rel }}$ is the energy relaxation rate in the electron gas and $A=L_{x} L_{y}$ is the cross-sectional area of the slab and $E_{z \text {,inside }}$ is the internal field normal to the slab walls (Fig. 5a). Note that the number of high-energy electrons does not depend on the slab width, $L_{N C}$. From Eq. (5), we see now three important properties: (1) the effect of photogeneration of high-energy carriers (electrons and holes) is a surface effect since it does not depend on the size of the slab, $\delta n(E) \sim L_{N C}^{0}$. (2) This effect weakens with increasing energy-relaxation rate, $\delta n \sim 1 / \gamma_{\text {rel }}$. (3) The effect is very sensitive to the direction of the exciting electric field, $\delta n(E) \sim\left|E_{z \text {,inside }}\right|^{2}$. Electrons with high excitation energies become generated only by the electric field normal to the wall.

To estimate the energy-relaxation rate, we adopted the experimental results from the time-resolved studies of gold nanocrystals [44] that give the energy relaxation time $\tau=0.5 \mathrm{ps}$ (also see Fig. 12 below). Therefore, for the relaxation rate we obtain $\gamma_{r e l}=/ \tau=1.3 \mathrm{meV}$. This relaxation rate describes the energy relaxation and thermalization of the Fermi gas and involves both phonons and electron-electron scattering. For simplicity, we do not involve here the radiative channel of relaxation assuming NCs of small sizes.

Since a confined system has a quantized spectrum, the calculated electron distribution exhibits steps due to the 2D electronic sub-bands in the slab (Fig. 5). In addition, we have performed an averaging of the electron spectra over the width of the slab in the interval $\left|L-L_{N C}\right|<\delta L=1 \mathrm{~nm}$, assuming the presence of variations of the slab thickness [45].

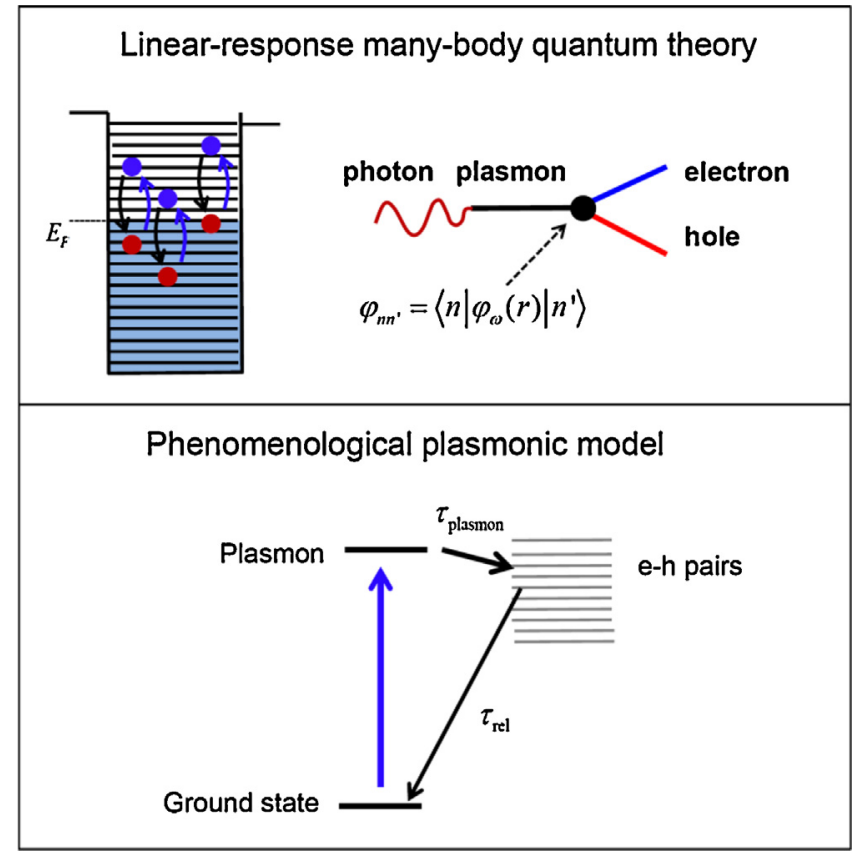

Figure 6 Illustrations of plasmonic processes within the linear-response and phenomenological models. The theoretical treatment of plasmonic dynamics in this paper is based on the linear-response many-body theory.

Phenomenological picture of plasmon dynamics

It is interesting to look at the plasmon kinetics using a phenomenological picture (Fig. 6). Within the linear-response many-body approach, the Fermi gas driven by the ac excitation field resides in a dynamic steady state (Fig. 6a). Electrons and holes are constantly being created and relaxing. In the quantum plasmonic picture, we should consider a plasmon as an excited quantum state of the electron system (Fig. 6, lower panel). Then, the plasmon undergoes fast de-phasing with creation of electron-hole pairs. Plasmon de-phasing comes from collisions with phonons and defects and also from electron-electron scattering [46]. The related plasmon coherence time is very fast, $\tau_{\text {plasmon }} \sim 9$ fs (see Section "Relaxation Mechanisms"). Qualitatively this coherence time of electron oscillations can be regarded as a plasmon lifetime. Consequently, the electron-hole pairs relax and thermalize further within the relaxation time $\tau_{\text {rel }}$. A balance equation for the plasmon population in a NC can be written as

$\frac{d n_{\text {plasmon }}}{d t}=\frac{\sigma_{a b s} I_{0}}{\omega_{p}}-\frac{n_{p l}}{\tau_{\text {plasmon }}}$,

where $n_{\text {plasmon }}$ is the number of plasmons created in the NC, and $\sigma_{a b s}$ and $I_{0}$ are the absorption cross section and light flux, respectively. In the steady state, $d n_{\text {plasmon }} / d t=0$ and the plasmon number $n_{\text {plasmon }}=\tau_{\text {plasmon }}\left(\sigma_{a b s} l_{0} / \omega_{p}\right)$. In relatively small NCs within the linear regime, the plasmon numbers are small, as expected. For example, $n_{p l} \sim 0.02$ for a $60 \mathrm{~nm}$ Au nanosphere in water, illuminated with $I_{0}=10^{4} \mathrm{~W} / \mathrm{cm}^{2}$ in the plasmon resonance. 


\section{Nanocrystals of various shapes}

Now we briefly consider nanocrystals of various shapes. So far, a full theory of photogeneration of plasmonic carriers has been developed only for the simplest geometry of a slab (or a platelet). However, this theory can be applied qualitatively to other shapes (nanospheres, nanocubes, nanorods, nanowires, etc.). In all these nanocrystals, an external electric field efficiently excites localized surface plasmons resonances and generates surface charges. Therefore, the electron currents in these geometries should hit the walls of these nanostructures and enable the creation of highenergy electrons. The key function for the plasmonic-carrier generation is the magnitude of the electric field inside the nanocrystal. The effect of generation of excited electrons comes from the plasmon-enhanced electric fields inside a NC.

The overall enhancement of the field inside a plasmonic NC can be conveniently quantified with the following function:

$F E_{N C}=\left(\int_{V_{N C}} \frac{\vec{E}_{\omega} \vec{E}_{\omega}^{*}}{E_{0}^{2}} d V\right)^{1 / 2}$

where $\vec{E}_{0}$ and $\vec{E}_{\omega}$ are the driving and internal electric fields, respectively. For the slab, sphere and ellipsoid geometries, this field-enhancement function can be calculated analytically. In particular, the function $F E_{N C}$ for a slab and a sphere is given by [47]

$P_{\text {slab }}(\omega)=\left|\frac{\varepsilon_{0}}{\varepsilon_{A u}}\right|, \quad P_{\text {sphere }}(\omega)=\left|\frac{3 \varepsilon_{0}}{2 \varepsilon_{0}+\varepsilon_{A u}}\right|$,

where $\varepsilon_{A u}$ and $\varepsilon_{0}$ are the dielectric constants of the metal and the matrix. In Fig. 7, we now plot the calculated field enhancements for the four types of NC. Nano-ellipsoids are obviously advantageous, due to a strong amplification of the field in the longitudinal plasmonic resonance.

\section{Application to photochemistry}

Plasmonic elections are being actively studied in experiments on photocatalysis [10-20]. Fig. 8 shows a simplified energy diagram for the transfer of plasmonic carriers to the external electron states. Such external electron states can be: (1) Molecules adsorbed on the metal surface, (2) a layer of material deposited on the surface, or (3) an electronic contact. This scheme is similar to that used for water splitting in the current experiments [9] (see further Fig. 14a in the section on experimental work). In this scheme, the energy of excited plasmonic electrons is transferred to the external states, creating reduced molecules or high-energy carriers in the external electrodes. Consequently, such energetic carriers or molecules can be used for photochemistry. This scheme has two energy parameters important for the extraction of electrons and holes from NCs: The barrier energies $\Delta \varepsilon_{e}$ and $\Delta \varepsilon_{h}$. These energies are calculated from the Fermi surface. Apparently, for efficient injection, we need to have sufficiently large numbers of electrons and holes at the energies $\Delta \varepsilon_{e}$ and $\Delta \varepsilon_{h}$. Such barrier energies are
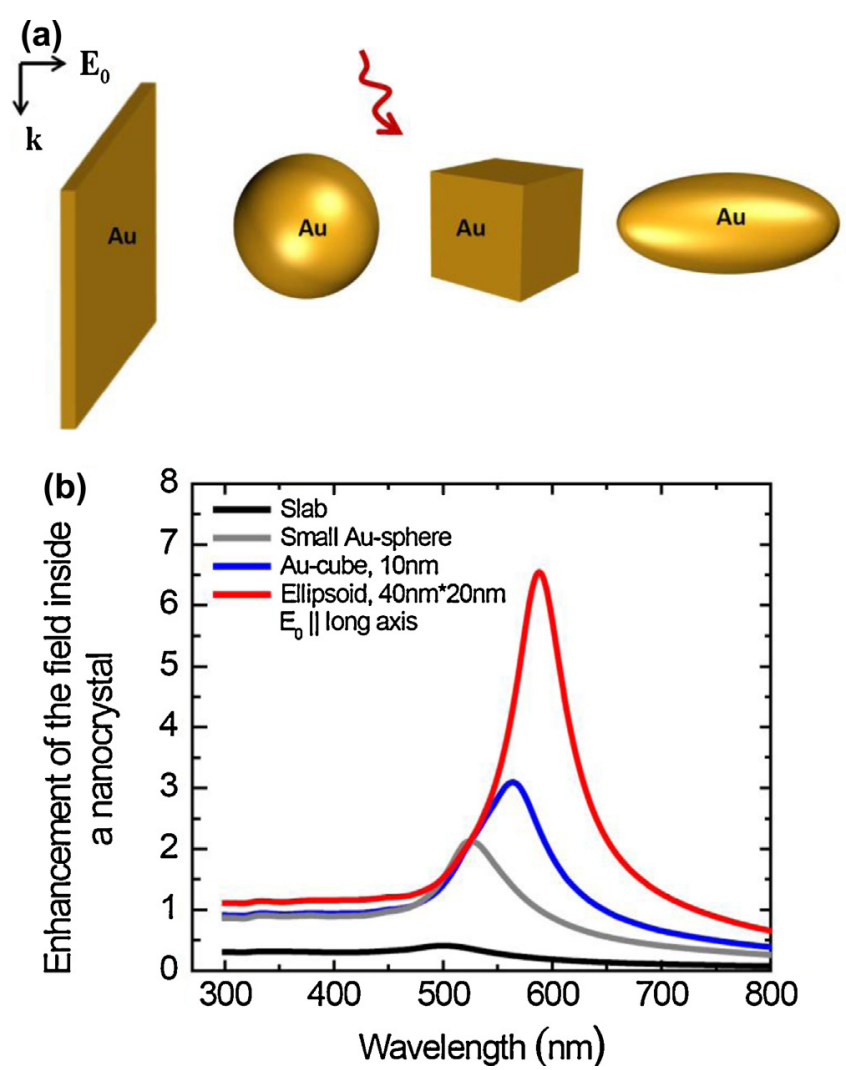

Figure 7 (a) Models of nanocrystals. (b) Calculated enhancement factors for the electric field of incident light inside $\mathrm{Au}$ nanocrystals of various shapes. Nanocrystals are assumed to be in a dielectric matrix (water).

typically $\sim \mathrm{eV}$. Therefore, the photons of visible light are able to generate carriers for injection, but the nanocrystal should be of a sufficiently small size. As we have seen in the previous section, only small NCs have a large number of highly excited carriers. The efficiency of generation of electrons (holes) with energy $\Delta \varepsilon_{e(h)}$ can be defined as

$E f f_{e(h)}\left(\omega, L_{N C}\right)=\frac{\text { Rate }_{e(h)}(\omega)}{\text { Rate }_{\text {absorption }}} \propto \frac{\left|\delta n\left(E_{F} \pm \Delta \varepsilon_{e(h)}, \omega, L_{N C}\right)\right|}{V_{N C}}$,

where Rate $_{\text {absorption }}$ is the rate of absorption of incident photons by a NC and Rate $_{e(h)}(\omega)$ is a rate of injection of electrons (holes) into the surface states. Here the electron injection rate is proportional to the population at the energy $E_{F}+\Delta \varepsilon_{e}$ : Rate Re $_{e}(\omega) \propto \delta n_{e}\left(E_{F}+\Delta \varepsilon_{e}\right)$. Similarly, for the holes, we have $\operatorname{Rate}_{h}(\omega) \propto-\delta n\left(E_{F}-\Delta \varepsilon_{e}\right)$. Simultaneously, the absorption rate Rate absorption $\propto V_{N C}$, where $V_{N C}$ is the $N C$ volume. Fig. 8 shows the calculated efficiency taken from Ref. [42]. The carrier generation efficiency decreases with increasing NC size since the energies required for injection are typically large, about $\mathrm{eV}$, and only relatively small NCs can generate efficiently such high-energy carriers. The number of energetic electrons (with $\Delta E \sim \mathrm{eV}$ ) is proportional to the surface area of the nanocrystal and, therefore, for a spherical or cubic nanoparticle

$E f f_{e(h)} \propto \frac{A_{N C}}{V_{N C}} \sim \frac{1}{L_{N C}}$ 
(a)

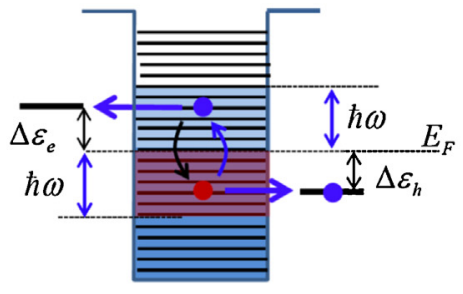

(c)

Absorption and hot-electron generation can be different

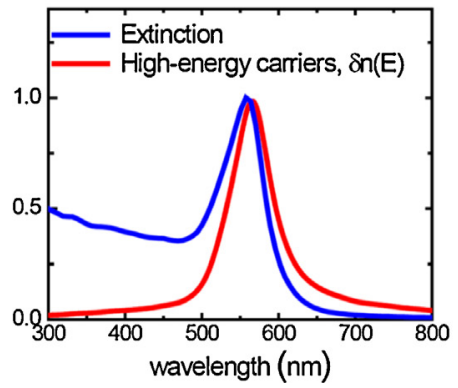

(b)

\section{Efficiency of hot-electron generation in a cubic nanocrystal}

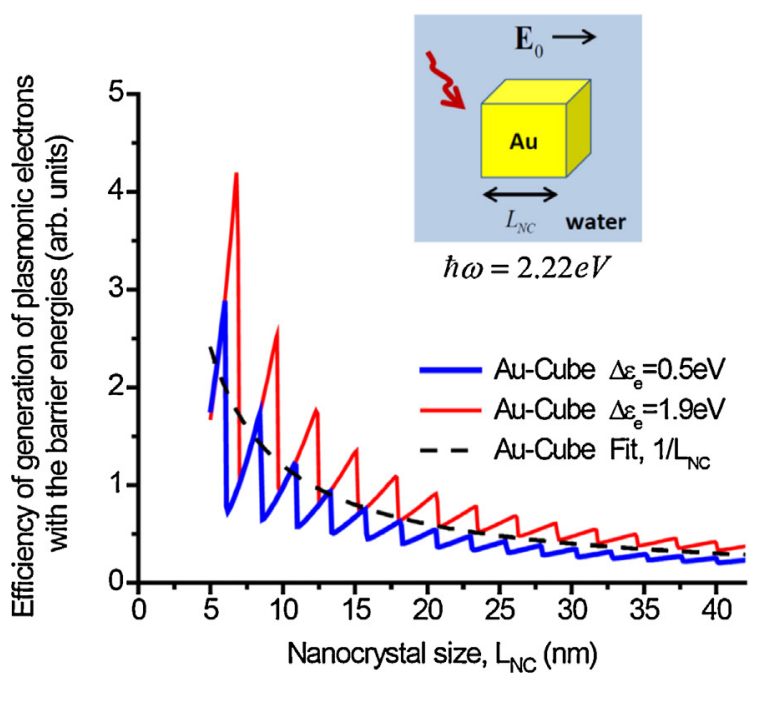

Figure 8 (a) Energy diagram for the transfer processes. (b) Calculated efficiencies of generation of plasmonic electrons with the barrier energies $E=E_{F}+\Delta \varepsilon_{e}$. Insert: Model of a cubic Au NC. Reprinted with permission from Ref. [42]. Copyright (2013) American Chemical Society. (c) Calculated number of high-energy electrons $\left(E-E_{F}=\Delta \varepsilon_{e}=1 \mathrm{eV}\right)$ and the absorption cross section, both normalized.

\section{Injection of plasmonic carriers into a semiconductor}

Another stream of research concerns injection of plasmonic carriers into semiconductors and organic materials [24-31]. One example is the semiconductor-metal Schottky contact with a barrier height $\Delta E_{B}$ (Fig. 9a). In general, the injection process depends on the boundary conditions at the interface between the metal and the semiconductor. For an ideal flat interface, an electron crossing the interface conserves its parallel momentum. Therefore, the conditions for injection of such electron should be written as

$E_{F}+\omega \geq E \geq E_{F}+\Delta E_{B}$ and $\frac{p_{z}^{2}}{2 m}>\Delta E_{B}+E_{F}$.

The first condition is the simple energy argument: The photoexcited electron should have energy above the barrier (Fig. 9a) while the energies of excited electrons are limited by $E_{F}+\omega$. The second one gives another restriction; this tells that the $z$-component of energy should be larger than the barrier height [49] (Fig. 9b). Physically, it means that, for a given energy, an electron can enter the contact only if its momentum is within the injection cone (Fig. 9b). Both conditions create restrictions for the numbers of electrons that can be injected into a contact. However, if the metal-semiconductor boundary is rough, the second condition can be removed and more electrons can be injected. In Fig. 9c and d, we show calculated rates of generation for a contact with a barrier $\Delta E_{B}=1 \mathrm{eV}$. We see the limitation coming from the conditions (6). First, the total rate of generation of plasmonic carriers exceeds the rate of generation of carriers above the barrier $E_{F}+\omega \geq E \geq E_{F}+\Delta E_{B}$.
However, the number of electrons that can enter the contact is even smaller because of the second restrictive condition, $p_{z}^{2} / 2 m>\Delta E_{B}+E_{F}$. As expected, the calculated curves in Fig. 9 exhibit oscillations owing to the size-quantization of electrons in a $20 \mathrm{~nm}$-thick metal slab. Fig. 9 illustrates the properties of a $1 \mathrm{eV}$ injection barrier, as an example. In real junctions, the barrier height varies in the range $0.5-1.5 \mathrm{eV}$ [3]. The experimental numbers for a few examples of Schottky barriers are given in Table 1. More data on the Schottky barriers can be found elsewhere [3].

The rate of injection at the threshold can be well approximated by the result from the Fowler theory [49]:

$I_{\text {photo-current }}=I_{0} \cdot\left(\omega-\Delta E_{B}\right)^{2}$

The quadratic Fowler law is based on the conditions (6). A full quantum mechanical solution to the problem of the photo-electric effect was given by Tamm [50] and further developed in Refs. [51,52]. The rate of injection from the full quantum treatment includes also the effect

Table 1 Data taken from Refs. [3,48].

\begin{tabular}{ll}
\hline Contact & Barrier height, $\Delta E_{B}=e \phi_{B}(\mathrm{eV})$ \\
\hline $\mathrm{n}-\mathrm{Si}-\mathrm{Au}$ & 0.83 \\
$\mathrm{p}-\mathrm{Si}-\mathrm{Au}$ & 0.34 \\
$\mathrm{TiO} 2-\mathrm{Au}$ & $0.87-0.94$ \\
$\mathrm{ZnO}-\mathrm{Au}$ & 0.65 \\
$\mathrm{GaAs}-\mathrm{Au}$ & $0.42-1.05$ \\
\hline
\end{tabular}


(a)

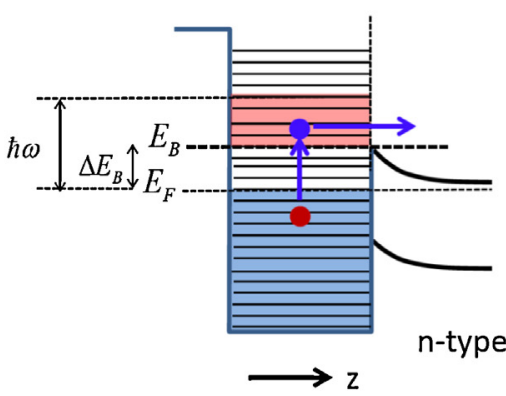

(c)

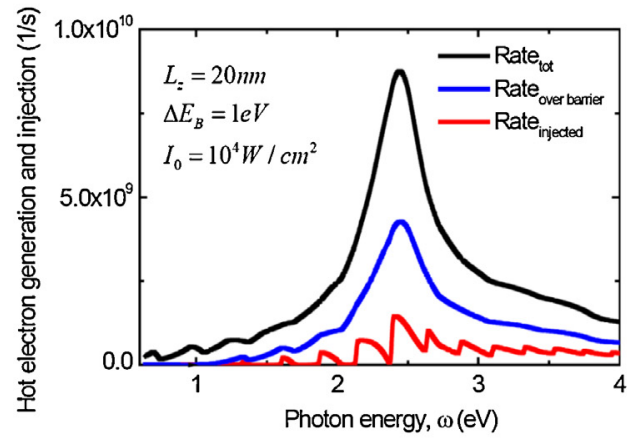

(b)

$E_{F}+\hbar \omega \geq E \geq E_{B}$

$\frac{p_{\mathrm{z}}^{2}}{2 m}>E_{B}$

$\mathbf{p}_{\|}$is conserved

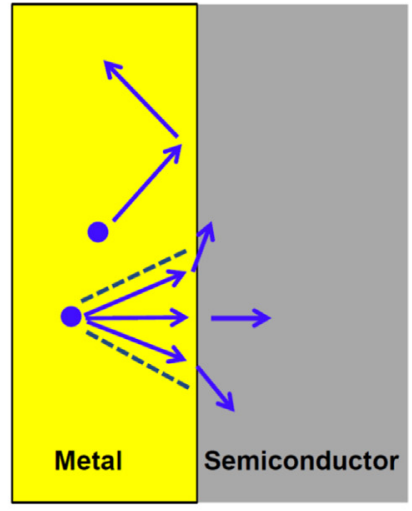

(d)

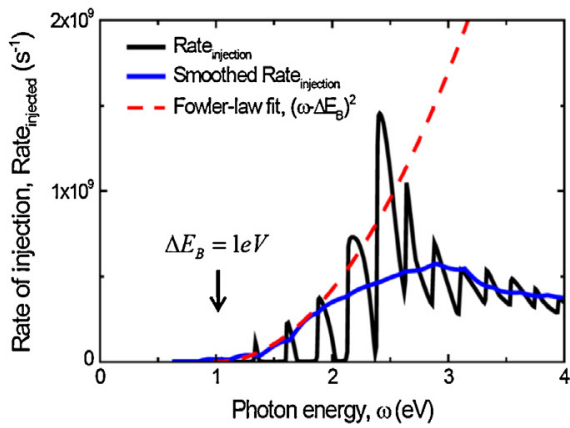

Figure 9 (a) and (b) Energy diagram and schematic of the Schottky barrier employed for injection of plasmonic electrons. (c) Calculated generation and injection rates for a plasmonic Au slab. (d) This graph shows closely the injection rate and its comparison with the Fowler law. The graphs (c) and (d) are reprinted with permission from Ref. [42]. Copyright (2013) American Chemical Society.

of over-barrier reflection of electrons and under certain conditions becomes:

$I_{\text {photo-current }}=I_{1} \cdot\left(\omega-\Delta E_{B}\right)^{5 / 2}$

Recently a theory of internal photo-effect has been further developed including several effects characteristic for the plasmonic systems. In particular, the recent studies concerned a reflection of electron waves in slabs $[53,54]$ and plasmonic effects in solar cells and photodetectors [55-57]. The quantum theory for surface photo-electric effect was used to calculate the photo-injection efficiencies in plasmonic nanostructures in Refs. [58,59]. A recent paper [60] described the non-equilibrium steady state of electrons in an optically driven metal nanoparticle using the energy balance equations.

\section{Band structure effects: inter-band and intra-band transitions}

The band diagram of a real metal is more complex than the spectrum of a free electron (Fig. 10a). For the case of $\mathrm{Au}$ and $\mathrm{Ag}$, the band structure can be understood in terms of $\mathrm{sp}$ - and d-bands. The sp-band resembles the free-electron dispersion and crosses the Fermi level. Transitions within the sp-band are intra-band excitations forming the Drude part of the dielectric constant. Optical transitions between the filled d-band and the empty states of the sp-band are inter-band excitations. We can see contributions of both types of transitions in the empirical dielectric function taken from Ref. [37] (Fig. 10b). A dielectric function of a bulk material can be written as a sum of two contributions, $\varepsilon_{\text {metal }}=\varepsilon_{\text {Drude }}+\varepsilon_{\text {inter-band }}$ [2]. The Drude contribution coming from the intra-band transitions has the form

$\varepsilon_{\text {Drude }}(\omega)=\varepsilon_{0}-\frac{\omega_{p}^{2}}{\omega\left(\omega+i \Gamma_{\text {bulk }}\right)}$

where the coefficients should be found from fitting to the empirical dielectric constant at long wavelengths (Fig. 10b). Indeed, we see that the Drude formula (7) gives an excellent fit to the experimental data, using $\Gamma_{\text {bulk }}=0.076 \mathrm{eV}$ and $\omega_{p}=8.9 \mathrm{eV}$ [62]. This indicates that gold behaves like a good metal. Then, we can see the contribution of the inter-band transitions by subtracting the Drude contribution from the empirical dielectric constant $\varepsilon_{\text {inter-band }}(\omega)=\varepsilon_{\text {empirical }}(\omega)-$ $\varepsilon_{\text {Drude }}(\omega)$. Since we now know the inter-band and intra-band contributions to the dielectric constant (Fig. 10b), we can calculate the corresponding components of absorption. The absorption in a NC can be written as a sum of two terms

$$
\begin{aligned}
Q_{\text {total }} & =Q_{\text {intra-band }}+Q_{\text {inter-band }}=2 \cdot \operatorname{Re} \int d V \quad\left(\mathbf{j} \cdot \mathrm{E}^{*}\right) \\
& =\frac{\omega}{2 \pi} \operatorname{Im}\left[\varepsilon_{\text {empirical }}(\omega)\right] \cdot \int d V \quad\left(\mathrm{E} \cdot \mathrm{E}^{*}\right) \\
& =\frac{\omega}{2 \pi} \operatorname{Im}\left[\varepsilon_{\text {Drude }}(\omega)+\varepsilon_{\text {inter-band }}(\omega)\right] \cdot \int d V \quad\left(\mathrm{E} \cdot \mathrm{E}^{*}\right),
\end{aligned}
$$


(a)

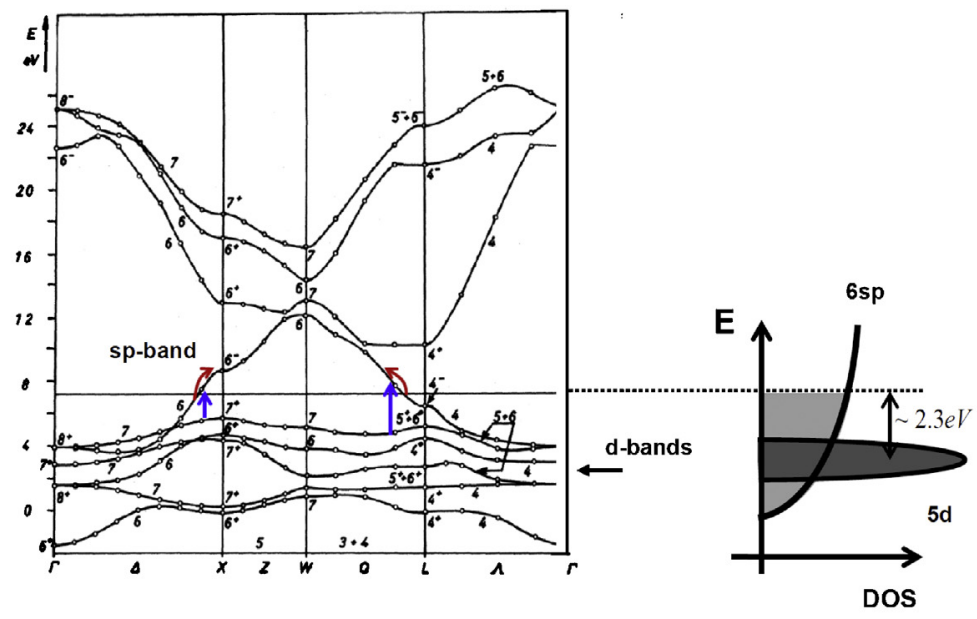

(b)

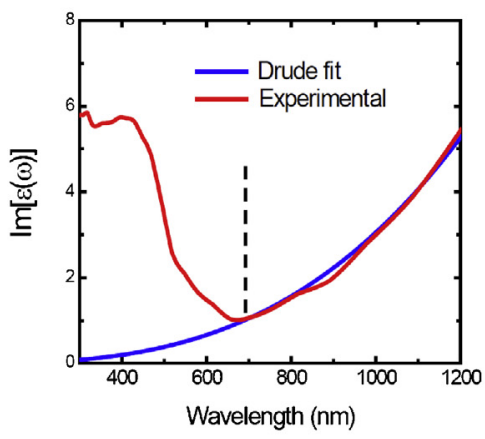

(c)

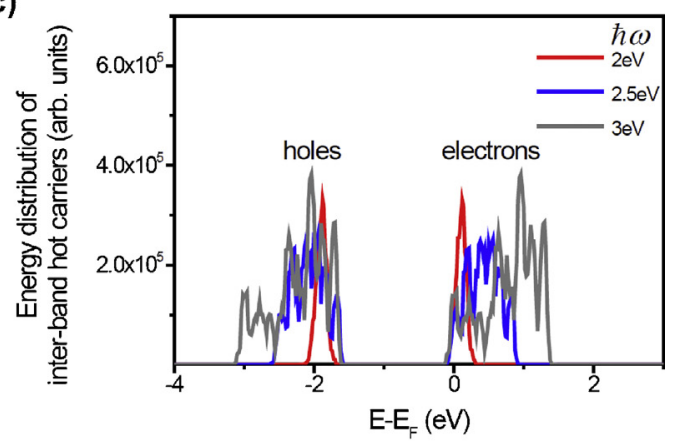

Figure 10 (a) Band diagram of gold, reproduced from Ref. [61]. We also show the intra-band and inter-band transitions (red and blue arrows). Copyright 1971 by The American Physical Society. Insert: Schematic of the density of states in the sp- and d-bands. (b) Imaginary part of the dielectric function of gold [37] and the Drude fit for the long wavelength limit. The difference between these two curves shows the presence of intensive inter-band transitions. The vertical line shows the transition between two regimes in the absorption; $\lambda<600 \mathrm{~nm}(\omega>2.07 \mathrm{eV})$ the imaginary part of the dielectric constant and the absorption are dominated by the inter-band transitions, while in this interval $\lambda>600 \mathrm{~nm}$ the intra-band Drude-like transitions govern the picture. (c) Distribution of hot plasmonic electrons and holes generated through the inter-band transitions in bulk gold. The calculation is done using the DFT theory.

where $\mathbf{j}$ and $\mathbf{E}$ are the complex amplitudes of electric current and electric field inside a NC, respectively. Fig. 11c shows the calculated contributions to the absorption for three different shapes. We see that the most common spherical NC has a small number of photogenerated intraband carriers and the majority of absorption in these NCs comes from the inter-band transitions. When we shift the plasmon resonance to the red, the contribution of intra-band transitions grows strongly. For structures with plasmon resonances longer than $600 \mathrm{~nm}$, the intra-band Drude-like absorption dominates. In the inset in Fig. 11c, we show the parameter $A$ for Au ellipsoid, which is defined as

$A=\frac{\int_{1.5 \mathrm{eV}}^{3 \mathrm{eV}} Q_{\text {intraband }} d \omega}{\int_{1.5 \mathrm{eV}}^{3 \mathrm{eV}} Q_{\text {total }} d \omega}$

We see a strong increase of the contribution from intraband transitions with increasing the aspect ratio $a / b$. This is because the plasmon resonance of an ellipsoid shifts to the red when the aspect ratio increases.

To summarize this section, we show qualitatively the spectrum of plasmonic electrons and holes in a NC excited with the photon energy $2.3 \mathrm{eV}$ (Fig. 11b). While the intra-band transitions produce energetic electrons and holes in the whole interval $E_{F}-\omega<E_{\text {excited electrons }}<E_{F}+$ $\omega$, the inter-band transitions (at the threshold photon energies $\omega \sim 2.1-2.4 \mathrm{eV}$ ) create mostly energetic holes with $E_{\text {excited holes }} \sim E_{F}-2.3 e V$ (Fig. 10c). Simultaneously, photogenerated electrons for such inter-band transitions are not energetic and have energies near the Fermi level, $E_{\text {excited electrons }} \sim E_{F}$.

\section{Relaxation mechanisms}

Relaxation mechanisms of electrons and plasmons are summarized in Fig. 12. The data on the mean-free path of excited electrons in gold can be found in Refs. [63,64]. The relaxation mechanisms of hot electrons involve electron-electron scattering and emission of phonons and photons $\left(\tau_{\text {rel }}=0.5-1 \mathrm{ps}\right)[44,46]$. These processes involve both intra-band and inter-band relaxations assisted by phonons, photons, and electron-electron scattering (Fig. 12a) [65]. In gold, optically generated electrons with excitation energies $1-2 \mathrm{eV}$ have mean-free path in the interval $17 \mathrm{~nm}<1_{\mathrm{mfp}}<50 \mathrm{~nm}$. This length determines the optimal sizes of plasmonic injectors. Devices with a shorter 
(a)

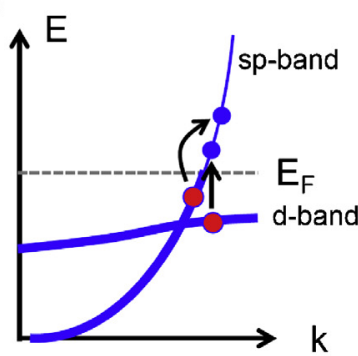

(b)

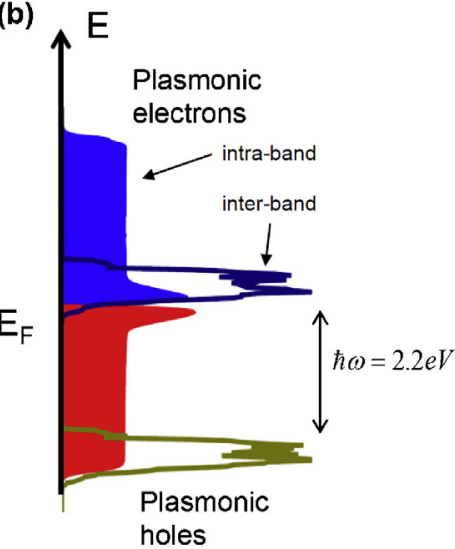

(c)
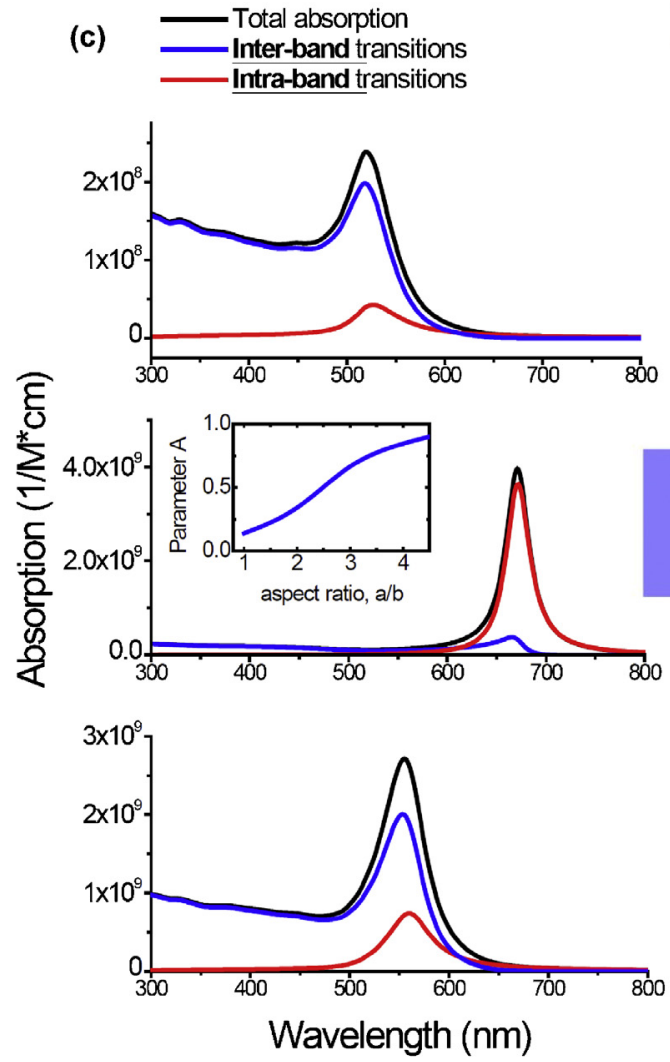
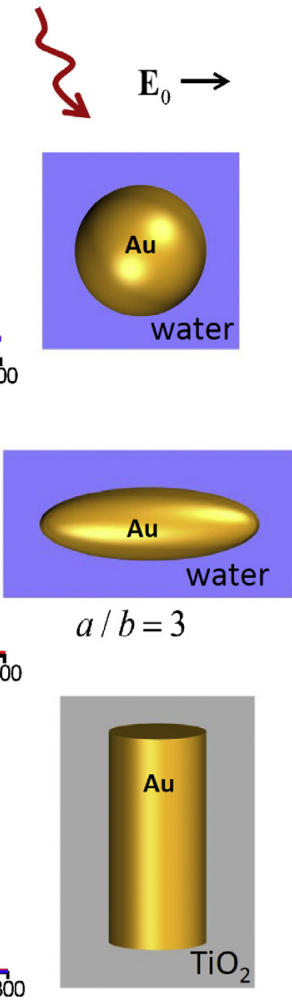

Figure 11 (a) Simplified diagram showing the inter-band and intra-band transitions in gold. (b) Distributions of plasmonic carriers created via the intra-band transitions (blue and red bands) and the distributions of carriers excited through the inter-band transitions (solid lines). As an example, the energy of exciting photons is taken as $2.2 \mathrm{eV}$. (c) Total absorptions and the contributions from the inter- and intra-band transitions. The calculations were carried out using the local dielectric function. Insets: Dielectric models of nanocrystals. Inset in the middle graph shows the ratio of intra-band and total absorptions for an Au ellipsoid as a function of the parameter $a / b$, where $a$ and $b$ are the sizes of ellipsoid.

dimension of $50 \mathrm{~nm}$ are the most advantageous since an energetic carrier in such a device can propagate to the surface without essential relaxation. Another possibility is to use high-quality metals with a long mean-free path, such as very high quality silver films fabricated in Ref. [66]. As we can see the mean free path of electron in gold is relatively short and the related lifetime is short as well, $l_{m f p} / v_{F} \sim 20 \mathrm{fs}$. This can be understood involving collisions with defects and phonons and also due to electron-electron scattering [67]. The fastest process in the collective dynamics is the de-phasing (coherent lifetime) of plasmon. This plasmon coherence time $\tau_{\text {plasmon }} \sim 5-10 \mathrm{fs}$ and can be estimated directly from the empirical dielectric function of gold as $\tau_{\text {plasmon }} \sim / \Gamma_{\text {bulk }} \sim 9 \mathrm{fs}$. The plasmon coherence life shown in Fig. $12 \mathrm{~d}$ is shorter due to the inter-band transitions that create an additional de-phasing mechanism for plasmons.

\section{Current experimental work and potential applications}

Here we briefly review some of the experimental reports in the field. The mechanisms responsible for plasmonenhanced photocurrents and photocatalysis can be divided into three groups: Thermally activated processes, processes amplified by the plasmon electromagnetic enhancement effect, and direct electron transfer/injection.

\section{Thermal effects and characteristic temperatures in the photo-excited metal nanocrystals}

One essential aspect of the problem is the photothermal effect in the plasmonic NCs [68,70-73]. Plasmonic NCs efficiently dissipate light energy, which may lead to an increase in local temperature of the system. This temperature increase can be a reason for the photocurrent generation [69], increased rates of chemical reactions [74,75], and photomelting of polymers and DNAs [76-78], plasmonic welding [79], etc. The increase in temperature at the surface of a single spherical nanoparticle derived from the thermal heat equation is

$\Delta T_{\text {single NC }}=\frac{Q_{\text {total }}}{4 \pi k_{0}} \frac{1}{R_{N C}}$

where $k_{0}$ is an averaged thermal conductivity of the surrounding matrix and $R_{N C}$ is a radius of heated NC. This temperature increase is typically small $(\sim 1-10 \mathrm{~K})$ for plasmonic NCs in solution illuminated with moderate light fluxes (Fig. 13a). However, a solution of weakly heated NCs can 


\section{Relaxation mechanisms in gold}
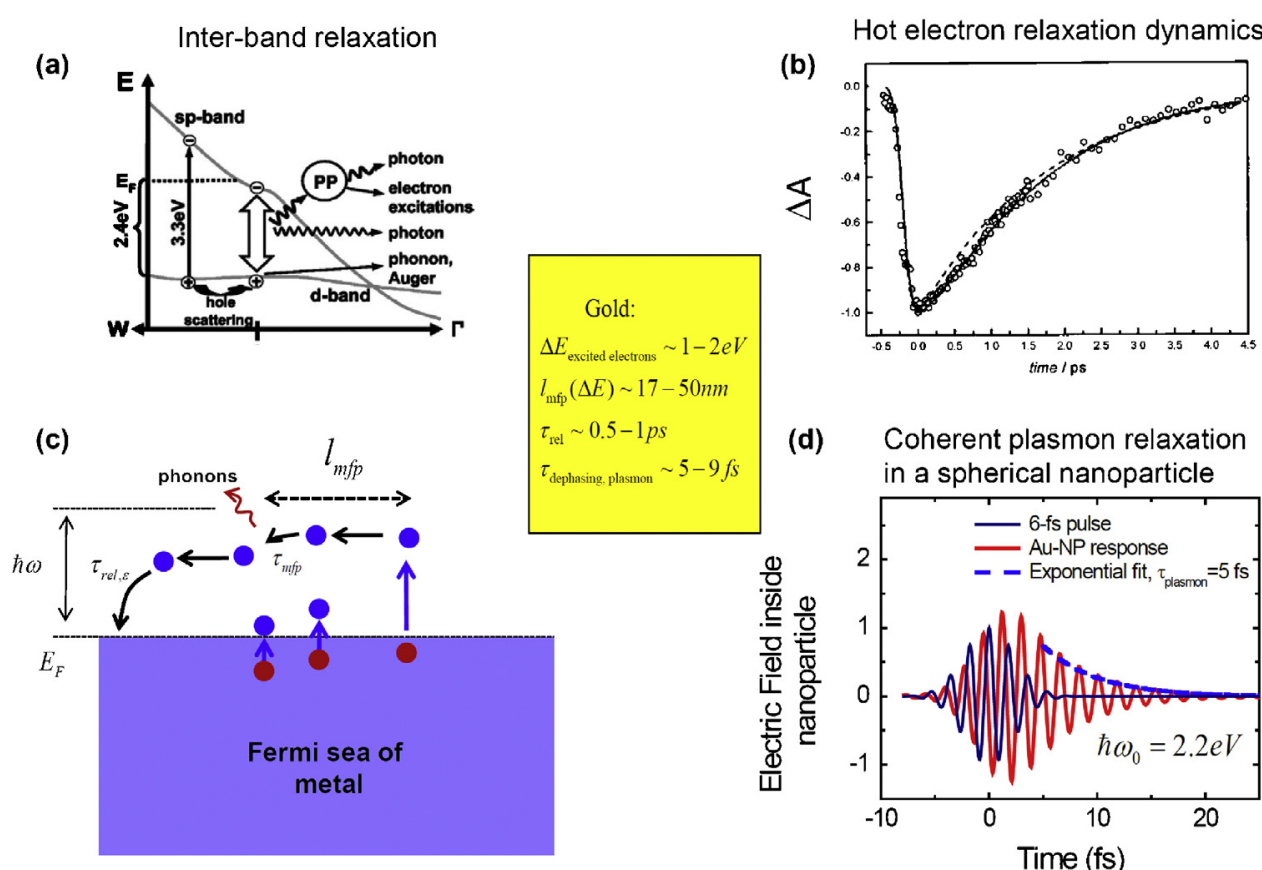

(d) Coherent plasmon relaxation in a spherical nanoparticle

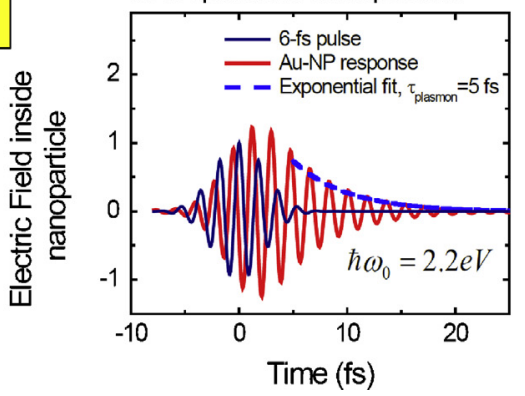

Figure 12 Review of relaxation mechanisms in plasmonic nanocrystals. (a) Inter-band relaxation of photogenerated carriers in gold. Taken from Ref. [65]. Copyright 2004 by The American Physical Society. (b) Dynamics of photobleaching of Au nanoparticles revealing the typical times of relaxation due to electron-electron and electron-phonon collisions. Reprinted with permission from Ref. [44]. Copyright (1999) American Chemical Society. (c) Illustration for the relaxation mechanisms and the parameters related to dynamics of photo-excited electron gas in a metal. (d) Calculated dynamics of relaxation of plasmonic electric field inside a plasmonic nanoparticle excited by a fs-pulse. This dynamics exhibits a short coherent lifetime of plasmon. The calculation was done using the local dielectric function of gold [37].

generate high temperatures due to the collective heating effect $[71,72,80]$. In this case, the resulting temperature increase can be large $[68,71]$,

$\Delta T_{\text {total }}=f\left(\rho_{N C}, R_{N C}\right) \cdot \Delta T_{\text {single } N C} \cdot N_{N C}^{2 / 3}$ where $N_{N C}$ is the number of NCs inside the volume of light beam and $\rho_{N C}$ is the NC density in solution. Since typically $N_{N C} \gg 1$, it is easy to realize the collective heating and boiling regimes when $\Delta T_{\text {total }} \gg \Delta T_{\text {single } N C}$ and $\Delta T_{\text {total }}$ can be above the boiling point. This effect can be used, for (a)

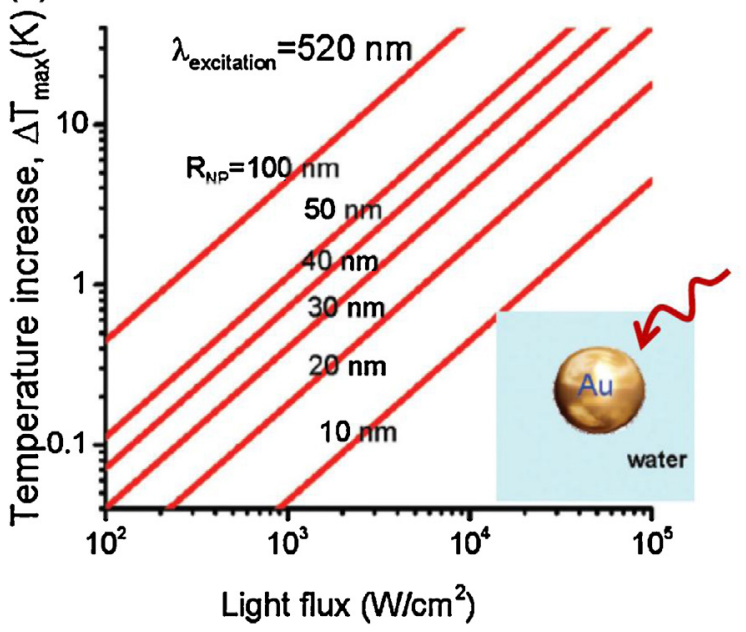

(b)
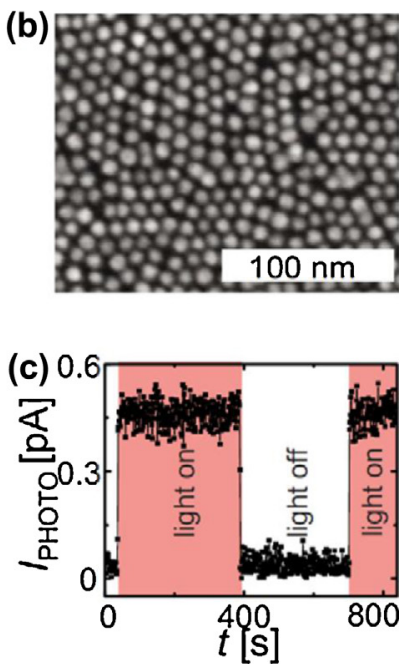

Figure 13 (a) Calculated temperature at the surface of a single Au nanoparticle in water. Reprinted from Ref. [68], Copyright (2006), with permission from Elsevier. (b) and (c) Monolayer of gold nanoparticles on a substrate and measured photocurrent in this system as a function of time. The origin of the photocurrent was identified as bolometric. Reprinted with permission from Ref. [69]. Copyright 2009, AIP Publishing LLC. 

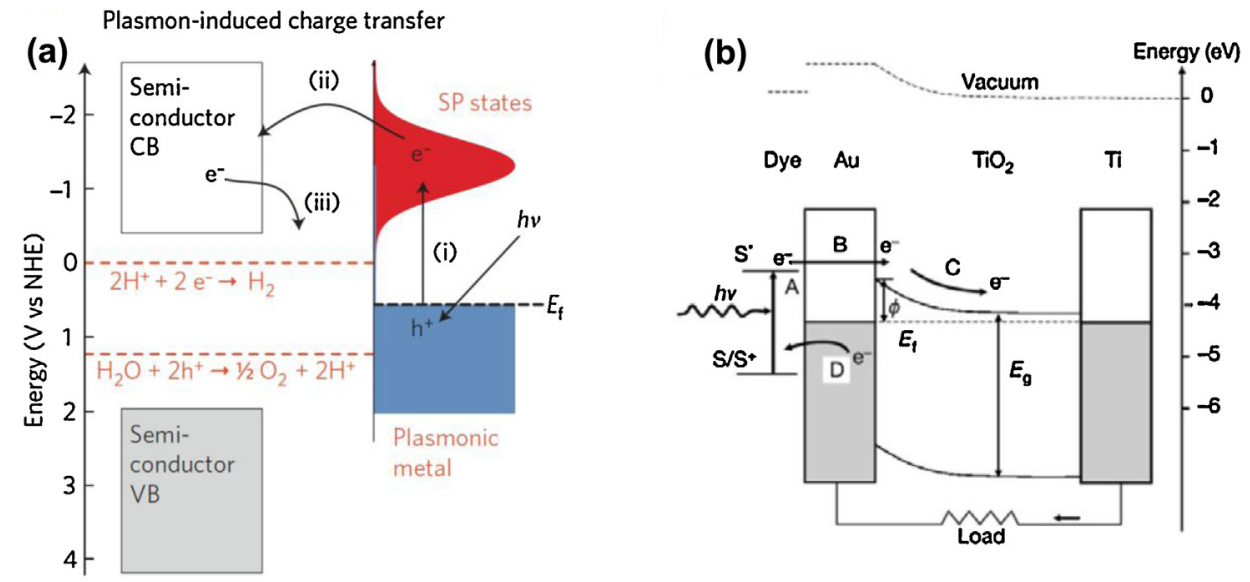

(c)
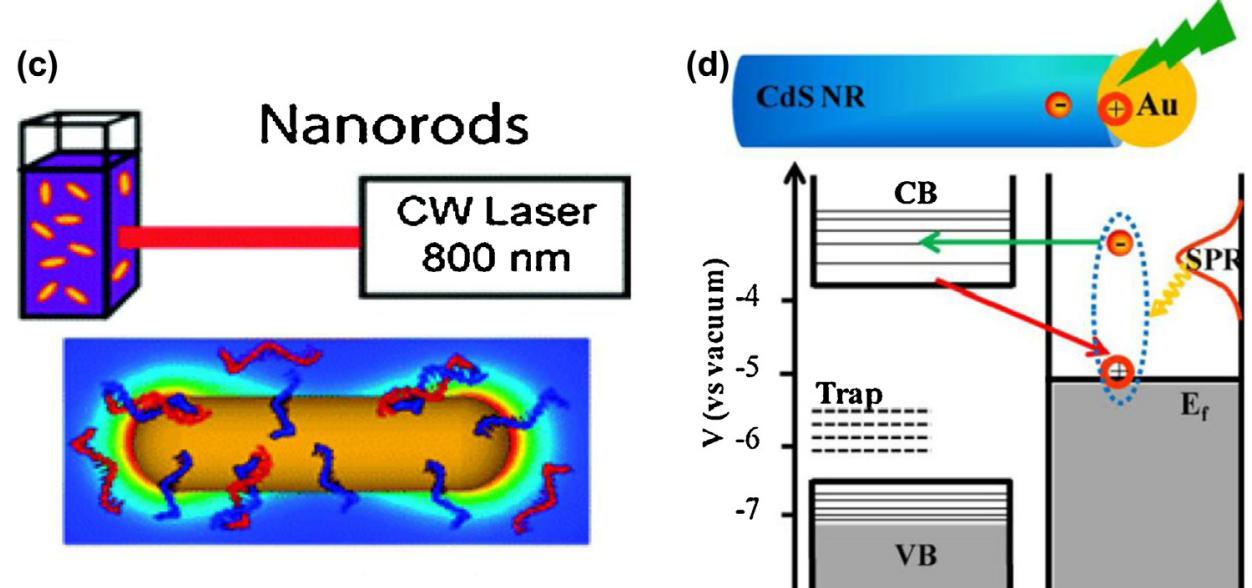

Figure 14 (a) Schematics of the water splitting reaction utilizing plasmonic carriers. Reproduced from Ref. [9]. Reprinted by permission from Macmillan Publishers Ltd: Nature Materials, copyright 2011. (b) The band diagram of a solar cell based on the optically-active adsorbed molecules and a metal film transmitting electrons. Taken from Ref. [82]. Reprinted by permission from Macmillan Publishers Ltd: Nature, copyright 2003. (c) Photo-triggered release of DNAs from plasmonic nanorods. Reprinted with permission from Ref. [14]. Copyright (2011) American Chemical Society. (d) Injection of plasmonic carriers from the Au nanoparticle to the semiconductor nanorod in a hybrid architecture. Reprinted with permission from Ref. [32]. Copyright (2013) American Chemical Society.

example, for boiling and thermal destruction of the matrix material $[76,81]$.

One of the first experiments on the photocurrents in plasmonic NC arrays is shown in Fig. 13b. A strong photocurrent response in this study was attributed and explained as a bolometric effect; the presence of hot electrons inside the NCs resulted in amplified electric currents through the junctions in the NC array.

\section{Elecromagnetic enhancement effects}

Plasmonic nanostructures are able to strongly enhance and trap electromagnetic fields and this effect offers interesting possibilities for designing more efficient light-harvesting system and solar cells [6,7]. Using plasmonic NCs and lithographically fabricated nanostructures, the electromagnetic field can be focused on the key regions of the system where photochemical transformations take place $[9,15,19,13]$. In particular, the study [19] presented hybrid nanocrystals in which the spatial area of photocatalytic activity (semiconductor nanoparticle) is separated from the plasmonic particle by a barrier; in this way it was demonstrated that the enhancement of chemical activity in this system comes from the plasmonic energy transfer, without direct transfer of carriers.

\section{Electron transfer and injection}

This direction is strongly motivated by the reports on photo-injection in purely solid-states devices incorporating a Schottky barrier and plasmonic waveguides and resonators $[24,25]$. Potential applications of such structures are in photo-detectors. Regarding solid-state and dye-sensitized solar cells, the metal component can be used as the main absorbing element [22] or as a mediator participating in electron and hole transfers [82]. The latter case is illustrated in Fig. 14b. In this system, the metal gate supplies electrons to the absorbing molecules and also transmits photoelectrons to the semiconductor. The other scheme is illustrated in Fig. 14a. In this case, the plasmonic nanocrystal 

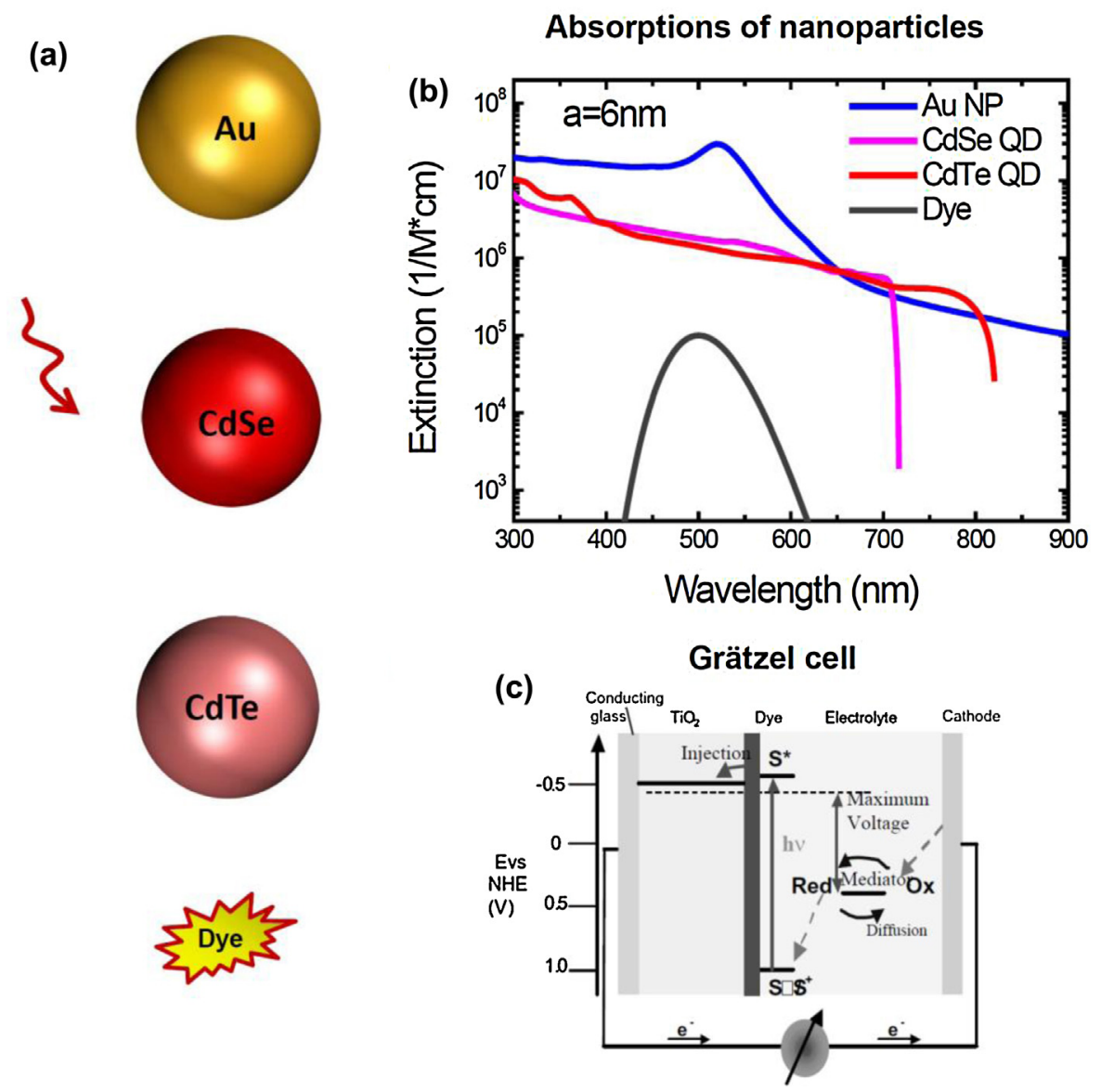

Figure 15 (a) Models of nanocrystal and molecular absorbers. (b) Absorptions calculated using Mie theory and local dielectric functions of the related materials; for the dye molecule, we took a typical extinction $10^{5}\left(\mathrm{Mcm}^{-1}\right.$. (c) Principle of operation and energy level scheme of the dye-sensitized nanocrystalline solar cell. Reprinted from Ref. [88], Copyright (2003), with permission from Elsevier.

is simultaneously an absorber of optical energy and a transmitter of carriers to a semiconductor and adsorbed molecules. At this moment, plasmonic photocatalysis has been used to drive several chemical reactions. Recent examples include dissociation of $\mathrm{O}_{2}[75,83]$ and $\mathrm{H}_{2}$ [21], splitting of water $[9,18,20,22,84]$, ethylene epoxidation [83], etc. Search for nanomaterials with a high efficiency of conversion of solar energy into chemical fuels is very active. Plasmonic electrons may also play an active role in bio-conjugated nanocrystals. For example, excited electrons of an optically driven NC and heating can trigger release of DNA molecules [14] (Fig. 14C). The mechanisms of generation and injection of plasmonic carriers are presently under active investigation using various spectroscopic techniques. In particular, a recent paper [32] has reported a time-resolved transmission study of electrons injected from an Au NC to a semiconductor nanorod. Another very recent paper [85] reported the experiment on focusing of surface plasmon polaritions on the metal-semiconductor junction using a tapered metal tip. In this system, the electron injection was enhanced by compressing the plasmon wave into a relatively small volume [86]. Interesting possibilities for hot electron injection and generation appear also in the photo-excited metal junctions under a strong electric bias when asymmetry of a junction is voltage-controlled [87].

\section{Conclusions}

This review article is mainly focused on the understanding of behaviors of excited electrons in optically driven metal nanocrystals. In the concluding Fig. 15, we show the absorption cross sections of various types of nanomaterials. The obvious advantage of plasmonic nanocrystals is in their strong absorption. Optical absorptions of metal NCs significantly exceed ones of semiconductor quantum dots, dye molecules and carbon nanotubes, which are used in dyesensitized solar cells [88] and other hybrid systems [89,90] (Fig. 15b). However, the challenge in the use of metal nanocrystals is in efficient extraction of the energetic electrons and holes. The lifetime of excited carriers in a metal is short, whereas excitons in dye molecules and semiconductor quantum dots are relatively long-lived. Other limiting factors for the use of plasmonic electrons are the transfer of momentum from a NC to plasmonic electrons and the reflection of carriers at interfaces. However, we show that the efficiency of generation and injection of plasmonic carriers can be increased by choosing appropriate sizes, geometries and excitation frequencies. We believe that this paper should be useful for understanding of generation and transport of hot plasmonic electrons in optically excited nanostructures. These optically active plasmonic systems 
can be used in a range of potential applications including optoelectronics, sensing, photochemistry and energy harvesting.

\section{Acknowledgments}

We thank Z. Fan for the DFT calculations of bulk gold. This work was supported by the Science Foundation Ireland (SFI $11 /$ W.1/12065 and SFI 07/IN.1/I1862 projects), the Ministry of Education and Science of the Russian Federation (Grant No. 14.B25.31.0002), the NSF (project: CBET-0933782), and Volkswagen Foundation. H.V.D. gratefully acknowledges support from NRF-RF-2009-09 and NRF-CRP-6-2010-2 as well as TUBA and ESF EURYI.

\section{References}

[1] S.A. Maier, Plasmonics: Fundamentals and applications, Springer, New York, 2007.

[2] L. Novotny, B. Hecht, Principles of Nano-Optics, Cambridge Univ. Press, Cambridge, UK, 2006.

[3] S.M. Sze, K.K. Ng, Physics of Semiconductor Devices, 3rd ed., Wiley, Hoboken, NJ, 2007.

[4] C.D. Lindstrom, X.-Y. Zhu, Chem. Rev. 106 (2006) 4281.

[5] C. Frischkorn, M. Wolf, Chem. Rev. 106 (2006) 4207.

[6] H.A. Atwater, A. Polman, Nat. Mater. 9 (2010) 205.

[7] J.A. Schuller, E.S. Barnard, W. Cai, Y.C. Jun, J.S. White, M.L. Brongersma, Nat. Mater. 9 (2010) 193.

[8] S.V. Boriskina, H. Ghasemi, G. Chen, Mater. Today 16 (2013) 375-386.

[9] S. Linic, P. Christopher, D.B. Ingram, Nat. Mater. 10 (2011) 911.

[10] C. Hubert, A. Rumyantseva, G. Lerondel, J. Grand, S. Kostcheev, L. Billot, et al., Nano Lett. 5 (2005) 615.

[11] L. Brus, Acc. Chem. Res. 41 (2008) 1742.

[12] X. Wu, E.S. Thrall, H. Liu, M. Steigerwald, L. Brus, J. Phys. Chem. C 114 (2010) 12896.

[13] Z. Liu, W. Hou, P. Pavaskar, M. Aykol, S.B. Cronin, Nano Lett. 11 (2011) 1111.

[14] R. Huschka, J. Zuloaga, M.W. Knight, L.V. Brown, P. Nordlander, N.J. Halas, J. Am. Chem. Soc. 133 (2011) 12247.

[15] I. Thomann, B.A. Pinaud, Z. Chen, B.M. Clemens, T.F. Jaramillo, M.L. Brongersma, Nano Lett. 11 (2011) 3440.

[16] M. Xiao, R. Jiang, F. Wang, C. Fang, J. Wang, J.C. Yu, J. Mater. Chem. 1 (2013) 5790.

[17] H. Tong, S. Ouyang, Y. Bi, N. Umezawa, M. Oshikiri, J. Ye, Adv. Mater. 24 (2012) 229.

[18] S.C. Warren, E. Thimsen, Energy Environ. Sci. 5 (2012) 5133.

[19] S. K. Cushing, J. Li, F. Meng, T.R. Senty, S. Suri, M. Zhi, et al., J. Am. Chem. Soc. 134 (2012) 15033.

[20] D.B. Ingram, S. Linic, J. Am. Chem. Soc. 133 (2011) 5202.

[21] S. Mukherjee, F. Libisch, N. Large, O. Neuman, L.V. Brown, J. Cheng, et al., Nano Lett. 13 (2013) 240.

[22] S. Mubeen, J. Lee, N. Singh, S. Krämer, G.D. Stucky, M. Moskovits, Nat. Nanotechnol. 8 (2013) 247.

[23] H.R. Stuart, D.G. Hall, Appl. Phys. Lett. 73 (1998) 3815.

[24] A. Akbari, R.N. Tait, P. Berini, Opt. Exp. 18 (2010) 8505.

[25] M.W. Knight, H. Sobhani, P. Nordlander, N.J. Halas, Science 332 (2011) 702.

[26] Y.K. Lee, C.H. Jung, J. Park, H. Seo, G.A. Somorjai, J.Y. Park, Nano Lett. 11 (2011) 4251.

[27] I. Goykhman, B. Desiatov, J. Khurgin, J. Shappir, U. Levy, Nano Lett. 11 (2011) 2219.

[28] M. Casalino, Int. J. Opt. Appl. 2 (2012) 1.

[29] D. Conklin, S. Nanayakkara, T.-H. Park, M.F. Lagadec, J.T. Stecher, X. Chen, et al., ACS Nano 7 (2013) 4479.
[30] F. Yan, X.W. Sun, Appl. Phys. Lett. 102 (2013) 043303.

[31] P. Berini, Laser Photon. Rev. (2013), http://dx.doi.org/ 10.1002/lpor.201300019.

[32] K. Wu, W.E. Rodríguez-Córdoba, Y. Yang, T. Lian, Nano Lett. 13 (2013) 5255.

[33] Y. He, P. Basnet, S. Murph, Y. Zhao, ACS Appl. Mater. Interfaces 5 (2013) 11818.

[34] E. Hao, G.C. Schatz, J. Chem. Phys. 120 (2004) 357.

[35] F.J.G. de Abajo, J. Phys. Chem. C 112 (2008) 17983.

[36] M. Liu, P. Guyot-Sionnest, T.W. Lee, S.K. Gray, Phys. Rev. B 76 (2007) 235428

[37] P.B. Johnson, R.W. Christy, Phys. Rev. B 6 (1972) 4370.

[38] P.M. Platzman, P.A. Wolff, Waves and Interactions in Solid State Plasma, Academic Press, New York, 1973.

[39] G.D. Mahan, Many-particle Physics, 3rd ed., Kluwer Academic/Plenum Publishers, New York, 2000.

[40] For gold, we can use $\varepsilon_{0}=9.01$ as the background dielectric constant, which describes the screening effect coming from the reaction of the core electrons of Au ions. In the reality, this is still a simplistic approximation for gold because of the presence of intensive inter-band transitions.

[41] A. Yariv, Quantum Electronics, 3rd ed., John Wiley \& Sons, New York, 1989.

[42] A.O. Govorov, H. Zhang, Y.K. Gun'ko, J. Phys. Chem. C 117 (2013) 16616.

[43] E. Devaux, T.W. Ebbesen, J.-C. Weeber, A. Dereux, Appl. Phys. Lett. 83 (2003) 4936.

[44] S. Link, M.A. El-Sayed, J. Phys. Chem. B 103 (1999) 8410.

[45] A.O. Govorov, H. Zhang, J. Phys. Chem. C (2014), http://dx. doi.org/10.1021/jp500009k.

[46] G.V. Hartland, Chem. Rev. 111 (2011) 3858.

[47] J.D. Jackson, Classical Electrodynamics, 3rd ed., John Wiley \& Sons, New York, 1998.

[48] N. Szydlo, R. Poirier, J. Appl. Phys. 51 (1980) 3310.

[49] R.H. Fowler, Phys. Rev. 38 (1931) 45.

[50] I. Tamm, S. Schubin, Z. Phys. 68 (1931) 97.

[51] K. Mitchel, Proc. R. Soc. Lond. 146 (1934) 442-464.

[52] A.M. Brodsky, Yu Ya. Gurevich, Sov. Phys. JETP 27 (1968) 114.

[53] C. Scales, P. Berini, IEEE J. Quant. Elect. 46 (2010) 633.

[54] Q.Y. Chen, C.W. Bates Jr., Phys. Rev. Lett. 57 (1986) 2737.

[55] T.P. White, K.R. Catchpole, Appl. Phys. Lett. 101 (2012) 073905.

[56] S. Zhu, G.Q. Lo, D.L. Kwong, Opt. Exp. 19 (2011) 15843.

[57] F.B. Atar, E. Battal, L.E. Aygun, B. Daglar, M. Bayindir, A.K. Okyay, Opt. Exp. 21 (2013) 7196.

[58] I.E. Protsenko, A.V. Uskov, Physics-Uspekhi 55 (2012) 508.

[59] A. Novitsky, A.V. Uskov, C. Gritti, I.E. Protsenko, B.E. Kardynat, A.V. Lavrinenko, Prog. Photovolt Res. Appl. (2012), http://dx.doi.org/10.1002/pip.2278.

[60] M. Kornbluth, A. Nitzan, T. Seideman, J. Chem. Phys. 138 (2013) 174707.

[61] N.E. Christensen, B.O. Seraphin, Phys. Rev. B 4 (1971) 3321.

[62] A. Vial, A.-S. Grimault, D. Macías, D. Barchiesi, M.L. de La Chapelle, Phys. Rev. B 71 (2005) 085416.

[63] C.R. Crowell, S.M. Sze, in: G. Hass, R.E. Thun (Eds.), Physics of Thin Films, Academic Press, 1967.

[64] C.R. Crowell, S.M. Sze, Phys. Rev. Lett. 15 (1965) 659.

[65] E. Dulkeith, T. Niedereichholz, T.A. Klar, J. Feldmann, G. von Plessen, D.I. Gittins, et al., Phys. Rev. B 70 (2004) 205424.

[66] Y.-J. Lu, J. Kim, H.-Y. Chen, C. Wu, N. Dabidian, C.E. Sanders, et al., Science 337 (2012) 450.

[67] K.W. Frese Jr., C. Chen, J. Electrochem. Soc. 139 (1992) 3234.

[68] A.O. Govorov, H.H. Richardson, Nano Today 2 (2007) 30.

[69] M.A. Mangold, C. Weiss, M. Calame, A.W. Holleitner, Appl. Phys. Lett. 94 (2009) 161104

[70] G.V. Hartland, Phys. Chem. Chem. Phys. 6 (2004) 5263.

[71] A.O. Govorov, W. Zhang, T. Skeini, H. Richardson, J. Lee, N. Kotov, Nanoscale Res. Lett. (First keynote issue) 1 (2006) 84. 
[72] P. Keblinski, D.G. Cahill, A. Bodapati, C.R. Sullivan, T.A. Taton, J. Appl. Phys. 100 (2006) 054305.

[73] G. Baffou, C. Girard, R. Quidant, Phys. Rev. Lett. 104 (2010) 136805.

[74] J.R. Adleman, D.A. Boyd, D.G. Goodwin, D. Psaltis, Nano Lett. 9 (2009) 4417.

[75] P. Christopher, H. Xin, S. Linic, Nat. Chem. 3 (2011) 467.

[76] A.G. Skirtach, C. Dejugnat, D. Braun, A.S. Susha, A.L. Rogach, W.J. Parak, et al., Nano Lett. 5 (2005) 1371.

[77] J. Stehr, C. Hrelescu, R.A. Sperling, G. Raschke, M. Wunderlich, A. Nichtl, et al., Nano Lett. 8 (2008) 619.

[78] A.S. Urban, M. Fedoruk, M.R. Horton, J.O. Rädler, F.D. Stefani, J. Feldmann, Nano Lett. 9 (2009) 2903.

[79] E.C. Garnett, W. Cai, J.J. Cha, F. Mahmood, S.T. Connor, M.G. Christoforo, et al., Nat. Mater. 11 (2012) 241.

[80] H.H. Richardson, Z. Hickman, A.O. Govorov, A. Thomas, W. Zhang, M.E. Kordesch, Nano Lett. 6 (2006) 783.

[81] D. Hühn, A.O. Govorov, P.R. Gil, W.J. Parak, Adv. Funct. Mater. 22 (2012) 294

[82] E.W. McFarland, J. Tang, Nature 421 (2003) 616.

[83] P. Christopher, H. Xin, A. Marimuthu, S. Linic, Nat. Mater. 11 (2012) 1044

[84] G. Wang, Y. Ling, H. Wang, X. Lu, Y. Li, J. Photochem. Photobiol. C Photochem. Rev. 19 (2014) 35.

[85] A. Giugni, B. Torre, A. Toma, M. Francardi, M. Malerba, A. Alabastri, et al., Nat. Nanotechnol. 8 (2013) 845.

[86] P.J. Schuck, Nat. Nanotechnol. 8 (2013) 799.

[87] A. Stolz, J. Berthelot, L. Markey, G. Colas des Francs, A. Bouhelier, (2013) arXiv:1308.4508.

[88] M. Grätzel, J. Photochem. Photobiol. C: Photochem. Rev. 4 (2003) 145.

[89] M.J. Berr, P. Wagner, S. Fischbach, A. Vaneski, J. Scheider, A.S. Susha, et al., Appl. Phys. Lett. 100 (2012) 223903.

[90] K.T. Dembele, G.S. Selopal, C. Soldano, R. Nechache, J.C. Rimada, I. Concina, et al., J. Phys. Chem. C 117 (2013) $14510-14517$.

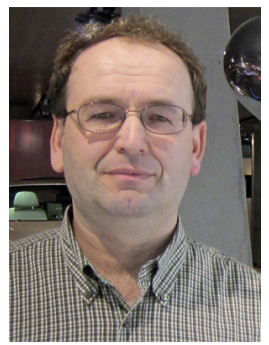

Alexander O. Govorov is Professor of Theoretical Physics at Ohio University in Athens, USA. PhD: 1991, Institute of Semiconductor Physics, Novosibirsk, Russia. Research position in the above Institute: 1987-2001. In 2001, he moved to the U.S. and became Professor at Ohio University. His research is focused on the theory of optical and electronic properties of nanostructures which incorporate semiconductor, metal, and molecular components. His theoretical predictions motivated experiments in many research labs worldwide. $\mathrm{He}$ is an author of more than 170 papers. He is a Fellow of the American Physical Society and a recipient of the Bessel Research Award (Humboldt Foundation, Germany), the Ikerbasque Research Fellowship (Spain), the E. T. S. Walton Visitor Award (Ireland), Chang Jiang Chair Professorship of the Scholar Program of MOE of China and 2014 Jacques-Beaulieu Excellence Research Chair Award (INRS, Montreal, Canada).

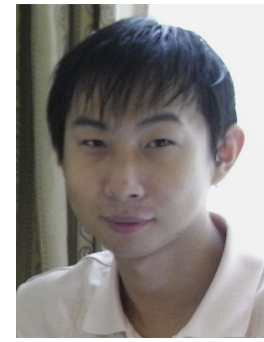

Hui Zhang received his B.Sc. degree in Applied Physics from the University of Science and Technology of China in 2006, and Ph.D. in Theoretical Physics from the Institute of Physics, Chinese Academy of Sciences in 2011. Currently, he is a postdoctoral researcher at the group of Prof. A. O. Govorov at Ohio University, U.S. His current research is on plasmonics and excitonics of hybrid nanostructures with a focus on quantum phenomena. He is an author of several recent papers published in Nano Letters, ACS Nano and other journals.

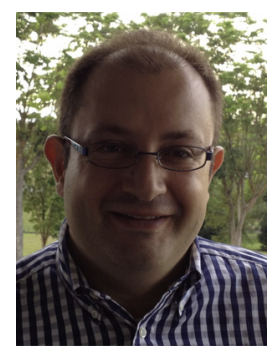

Hilmi Volkan Demir is a professor of electrical engineering and physics. He received his M.S. and Ph.D. degrees at Stanford University. He was named a Fellow by the Singapore National Research Foundation and was also appointed to a Nanyang Associate Professorship at NTU Singapore. Concurrently, he is the EURYI Associate Professor at Bilkent University, Turkey. He serves as the Director of the Luminous! Center of Excellence. Among his research interests are light-matter interactions at the nanoscale and semiconductor device physics. Dr. Demir has contributed to commercialization and licensing of several new enabling technologies as well as establishing a successful company and led to $>20$ patent applications, several of which have currently been used, owned or licensed by the industry. These scientific and entrepreneurship activities resulted in several important international awards including European Science Foundation European Young Investigator Award and The Outstanding Young Person in the World Award by JCl Federation of Young Leaders and Entrepreneurs.

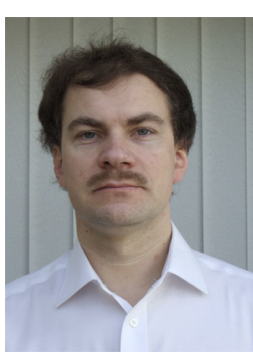

Yurii Gun'ko graduated from the Chemistry Department of Moscow State University in 1987. He has also received his Ph.D degree in Inorganic Chemistry from Moscow State University in 1990. Then he worked as a lecturer in Chemistry in Belorussian Institute of Technology (Belarus). In 1994 he received a postdoctoral position in the group of professor M.F. Lappert in the University Sussex (UK). Then in 1995 he was awarded Alexander von Humboldt fellowship and worked in the University of Magdeburg (Germany) with professor F. T. Edelmann. After that he returned back to the University Sussex and worked where as a postdoctoral researcher. In 1999 Dr. Gun'ko moved to the Chemistry Department of Trinity College Dublin (Ireland) to take up the position of the lecturer in Inorganic Chemistry. Currently Yurii Gun'ko works as a Professor in the School of Chemistry and a Principal Investigator in CRANN Institute in Trinity College. His main research interest and activities are: carbon nanomaterials, photovoltaic cells, magnetic nanostructures, plasmonic and quantum dot based materials. 